Pacific Northwest

National Laboratory

Operated by Battelle for the

U.S. Department of Energy

\title{
Modeling LIDAR Detection of Biological Aerosols to Determine Optimum Implementation Strategy
}

DM Sheen

PM Aker

September 2007

Prepared for the U.S. Department of Energy under Contract DE-AC05-76RL01830 


\title{
DISCLAIMER
}

This report was prepared as an account of work sponsored by an agency of the United States Government. Neither the United States Government nor any agency thereof, nor Battelle Memorial Institute, nor any of their employees, makes any warranty, express or implied, or assumes any legal liability or responsibility for the accuracy, completeness, or usefulness of any information, apparatus, product, or process disclosed, or represents that its use would not infringe privately owned rights. Reference herein to any specific commercial product, process, or service by trade name, trademark, manufacturer, or otherwise does not necessarily constitute or imply its endorsement, recommendation, or favoring by the United States Government or any agency thereof, or Battelle Memorial Institute. The views and opinions of authors expressed herein do not necessarily state or reflect those of the United States Government or any agency thereof.

\author{
PACIFIC NORTHWEST NATIONAL LABORATORY \\ operated by \\ BATTELLE \\ for the \\ UNITED STATES DEPARTMENT OF ENERGY \\ under Contract DE-AC05-76RL01830
}

Printed in the United States of America
Available to DOE and DOE contractors from the
Office of Scientific and Technical Information,
P.O. Box 62, Oak Ridge, TN 37831-0062;
ph: (865) 576-8401
fax: (865) 576-5728
email: reports@adonis.osti.gov

\begin{abstract}
Available to the public from the National Technical Information Service, U.S. Department of Commerce, 5285 Port Royal Rd., Springfield, VA 22161 ph: (800) 553-6847 fax: (703) 605-6900

email: orders@ntis.fedworld.gov

online ordering: http://www.ntis.gov/ordering.htm
\end{abstract}

This document was printed on recycled paper. 


\title{
Modeling LIDAR Detection of Biological Aerosols to Determine Optimum Implementation Strategy
}

\author{
D. M. Sheen \\ P. M. Aker
}

September 2007

Prepared for

the U.S. Department of Energy

under Contract DE-AC05-76RL01830

Pacific Northwest National Laboratory

Richland, Washington 99352 



\section{Summary}

This report summarizes work performed for a larger multi-laboratory project named the Background Interferent Measurement and Standards project. While PNNL was originally tasked to develop algorithms to optimize biological warfare agent detection using UV fluorescence LIDAR, the current uncertainties in the reported fluorescence profiles and cross sections preclude the development of any meaningful models. It was decided that a better approach would be to model the wavelength-dependent elastic backscattering from a number of ambient background aerosol types, and compare this with that generated from representative sporulated and vegetative bacterial systems. Calculations in this report show that a 266, 355, 532, and 1064 nm elastic-backscatter LIDAR experiment will allow an operator to immediately recognize when sulfate, VOC-based, or road dust (silicate) aerosols are approaching, independent of humidity changes. It will be more difficult to distinguish soot aerosols from biological aerosols, or vegetative bacteria from sporulated bacteria. In these latter cases, the elastic scattering data will most likely have to be combined with UV fluorescence data to enable a more robust categorization. 


\section{Acknowledgments}

This work was funded through the U.S. Army’s West Desert Test Center at the Dugway Proving Ground with support from the Defense Threat Reduction Agency. 


\section{Glossary}

$\begin{array}{ll}\text { BSAS } & \text { Bio-Spectral Algorithm Stimulator } \\ \text { CCN } & \begin{array}{l}\text { cloud condensation nuclei } \\ \text { CMD }\end{array} \\ \text { DPA } & \text { dipicolinic acid } \\ \text { GUI } & \text { graphical user interface } \\ \text { IR } & \text { infrared } \\ \text { JBSDS } & \text { Joint Biological Standoff Detection System } \\ \text { LIDAR } & \begin{array}{l}\text { Light Detection and Ranging } \\ \text { LIF }\end{array} \\ \text { MMD } & \text { maser-induced fluorescence } \\ \text { NAD(P)H } & \text { nicotinamide adenine dinucleotide phosphate } \\ \text { RH } & \text { relative humidity } \\ \text { SAS } & \text { Spectral Algorithm Stimulator } \\ \text { SMD } & \text { surface median diameter } \\ \text { UV } & \text { volatile organic chemical } \\ \text { VOC } & \end{array}$




\section{Contents}

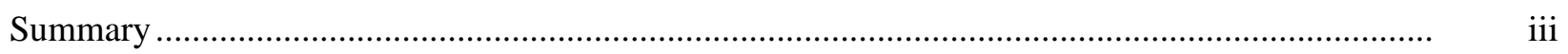

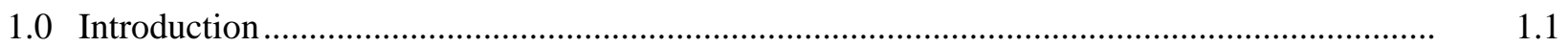

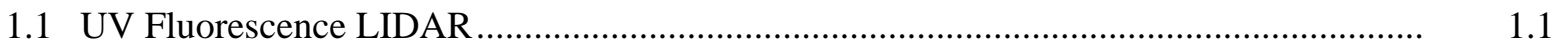

1.2 Multiple-Wavelength Elastic Backscatter LIDAR .......................................................... 1.3

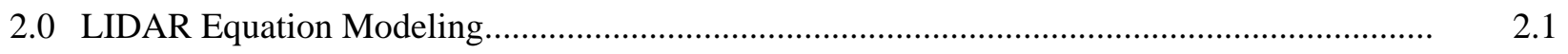

3.0 Aerosol Modeling for Biological Particles......................................................................

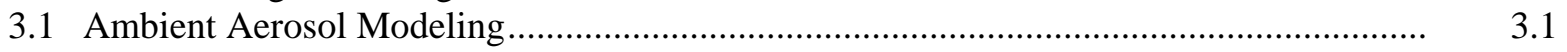

3.1.1 Rayleigh Molecular Scattering Model ............................................................ 3.1

3.1.2 Natural Aerosol Scattering Model.................................................................... 3.3

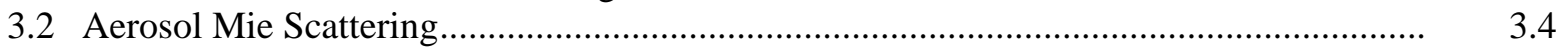

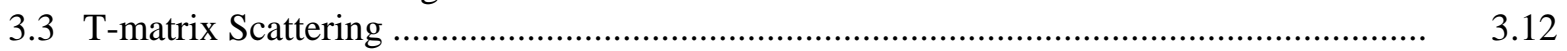

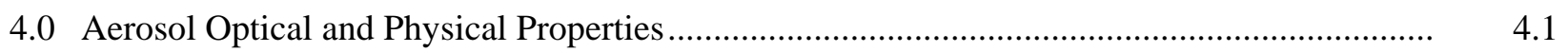

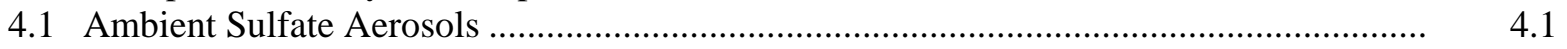

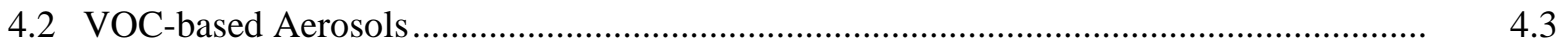

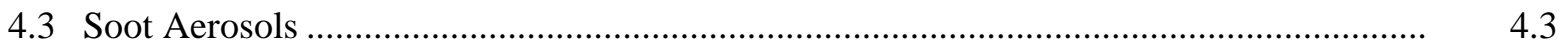

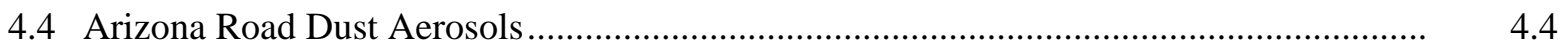

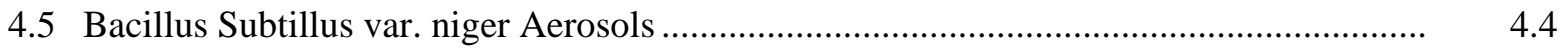

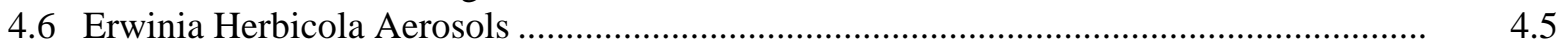

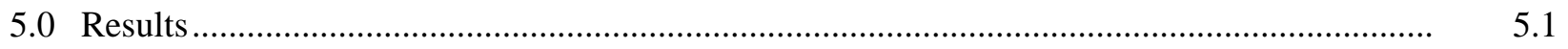

5.1 Influence of Humidity on Ambient Aerosol Scattering .................................................. 5.1

5.2 Elastic Backscattering Profiles of Biological and Non-biological Aerosols ...................... 5.2

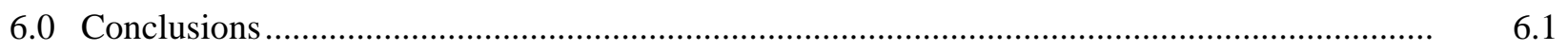

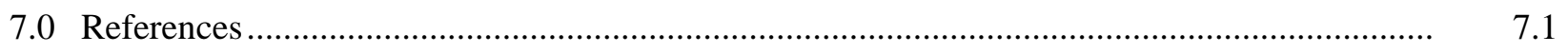

Appendix A - Modeling Code Description - MatLab .......................................................... A.1 


\section{Figures}

1.1 Measured Wavelength Dependence of (a) Real and (b) Imaginary Parts of the

Refractive Index of Various Aerosol Components ...

3.1 Spherical Dielectric Particle Illuminated by a Plane Optical Wave.

3.2 Mie Efficiencies for a Low-Loss Case with $m=1.4+0.005 i$

3.3 Mie Efficiencies for a Medium-Loss Case with $m=1.4+0.05 i$.

3.4 Mie Efficiencies for a High-Loss Case with $m=1.4+0.5 i$

3.5 Mie Backscattering Efficiency in the Small Sphere (Rayleigh) Limit

3.6 Mie Backscattering Efficiency in the Large Sphere Limit

3.7 Mie Extinction Efficiency in the Large Sphere Limit.

5.1 Elastic Backscatter Cross Sections Calculated for Sulfate and VOC Aerosol Systems as a Function of Relative Humidity

5.2 Elastic Backscatter Cross Sections for the Different Aerosol Systems.

5.3 Wavelength Dependence of Relative Elastic Backscatter Cross Sections

\section{Tables}

5.1 Calculated Elastic Backscatter Cross Sections in $\mu m^{2}$. 


\subsection{Introduction}

It has been recognized that a number of hostile states and terrorist groups have intentions to use biological warfare agents to attack U.S. personnel and infrastructure both at home and abroad. As a result, the Departments of Defense and Homeland Security have invested heavily in developing a number of different biological warfare agent sensors. One sensor that has received considerable attention is the Joint Biological Standoff Detection System (JBSDS) (NRC 2005). JBSDS developers (represented by several independent groups) have claimed that Light Detection and Ranging (LIDAR) technology can be adapted to be able to detect and discriminate a biological agent aerosol mass from ambient aerosol clouds at safe stand-off distances. In its current form, the JBSDS uses two LIDAR systems, one in the shortwave (or near) infrared (IR) and another in the ultraviolet (UV), to look for and categorize approaching aerosol masses.

LIDAR involves sending laser light out from a pulsed source and analyzing the resulting backscattered (i.e., returned) light to quantify some property of the atmosphere. When light is scattered elastically (i.e., no change in wavelength) off molecules found in the atmosphere, the process is known as Rayleigh scattering; elastic scattering off particulate matter (aerosols) is known as Mie scattering. It is well established that IR LIDAR is an effective tool for detecting ambient aerosols in both the troposphere and stratosphere. Here the amplitude of the return signal is proportional to the number of aerosol particles present (and their size, shape, and complex refractive index); and the time delay and pulse width of the return signal yields the distance and depth of the cloud, respectively. It is important to note that while single-wavelength IR LIDAR return signals can warn of an aerosol cloud's presence, it cannot tell what types of aerosols make up the cloud. In the JBSDS case, the IR LIDAR is used only to warn the user that an aerosol cloud is approaching. Because there is very little atmospheric absorption in the shortwave IR, the IR LIDAR can detect potential threat clouds out to long distances, up to $20 \mathrm{~km}$ in some cases.

\subsection{UV Fluorescence LIDAR}

The JBSDS also uses UV laser-induced fluorescence LIDAR wavelengths to try to characterize the suspect aerosol cloud. The ranging capability of this system is much shorter, $1 \mathrm{~km}$ or less, than seen with IR LIDAR because ambient atmospheric molecules absorb quite strongly in the UV (meaning that the incident beam becomes attenuated quite rapidly) and sunlight (or moonlight) contributes a high UV background that leads to increased noise on the return signal. UV fluorescence LIDAR is a simple extension of UV laser-induced fluorescence; i.e., when the wavelength of a probe laser is coincident with electronic absorptions of species contained within an aerosol, some of the laser energy will be absorbed and then reemitted at a different wavelength. This fluorescence can be used to characterize the chemical composition of the aerosol.

JBSDS developers have posited that UV fluorescence LIDAR can be used to distinguish biological aerosols from non-biological aerosols. This is because biological systems contain a number of species that have characteristic UV fluorescence profiles. Some early versions of the JBSDS UV-laser-induced fluorescence (LIF) LIDAR used an excitation source at $280 \mathrm{~nm}$ and measured fluorescence returns at $350 \mathrm{~nm}$. The rationale for choosing a $280-\mathrm{nm}$ source was based on a conventional UV-LIF analytical technique that was developed to measure protein concentrations. The amino acids tyrosine and tryptophan are present in the cell material of all biological organisms and the cell wall of bacterial spores. 
Both absorb energy between 260 and $280 \mathrm{~nm}$. The fluorescence signal seen from tryptophan, which has an absorption maximum at $280 \mathrm{~nm}$, spans a region between 300 and $450 \mathrm{~nm}$ with the peak emission intensity at $350 \mathrm{~nm}$ (Fell et al. 1998; Halverson et al. 2003). Tyrosine fluoresces between 280 and $400 \mathrm{~nm}$ with the peak at $310 \mathrm{~nm}$ (Hill et al. 1999; Pan et al. 1999; Halverson et al. 2003). Of the two acids, tryptophan has the largest absorption cross section and is expected to be the major component of fluorescence in biological systems. It is worthwhile to note that short-wavelength (260-280 nm) excitation LIF cannot specifically distinguish harmful biological agents from other biological material such as pollen grains or other innocuous bacteria or spores. The fluorescence response at $350 \mathrm{~nm}$ after excitation between $260-280 \mathrm{~nm}$ only means that an aerosol/particulate with the protein tryptophan is probably present. It is does not indicate that the aerosol contains live agents, nor even if the aerosol consists of vegetative bacteria or spores.

It has been noted that, in addition to emission from amino acids, there are other sources of fluorescence that could be used to categorize biological aerosols. Nicotinamide adenine dinucleotide has an absorption peak at $340 \mathrm{~nm}$ and a broadband fluorescence that peaks at $470 \mathrm{~nm}$ (Fell et al. 1998; Hill et al. 1999; Halverson et al. 2003). The fact that the reduced form of nicotinamide adenine dinucleotide phosphate, $\mathrm{NAD}(\mathrm{P}) \mathrm{H}$, absorbs at a different wavelength from the oxidized form, $\mathrm{NAD}(\mathrm{P})$, provides a potential method for distinguishing between viable and non-viable bacteria. When bacteria die, they convert to the oxidized state. Therefore, the differential between NAD $(\mathrm{P}) \mathrm{H}$ and $\mathrm{NAD}(\mathrm{P})$ can provide viability information in near-real time. Another indicator of biological activity is riboflavin. Riboflavin absorption peaks at $385 \mathrm{~nm}$ and its broadband emission peaks at $525 \mathrm{~nm}$ (NRC 2005). And with excitation at $360 \mathrm{~nm}$, observance of $440 \mathrm{~nm}$ peak fluorescence from dipicolinic acid (DPA) can be used to discriminate between vegetative and sporulated bacteria (Nudelman et al. 2000; Sarasanandarajah et al. 2005a). Research is currently underway to determine if adding these additional excitation wavelengths will enhance the ability to determine viability at the same time as discriminating biological spores from vegetative bacteria or non-biological particles in the environment. It is worthwhile to note that adding the longer wavelength excitation - 355 nm for $\mathrm{NAD}(\mathrm{P}) \mathrm{H}$ and DPA and $400 \mathrm{~nm}$ for riboflavin - extends the daytime range of a UV fluorescence LIDAR system because atmospheric attenuation is weaker here than at $266 \mathrm{~nm}$.

Despite the promise of using UV fluorescence LIDAR to categorize aerosols as biological or nonbiological, viable or non-viable, or vegetative versus sporulated, little progress has been made in developing this technology as a fieldable stand-off biological agent detector. There are several reasons for this lack of progress. First, there are conflicting reports on the capability of being able to use wavelength-resolved fluorescence (or excitation-emission measurements) to identify different classes of biological agents, or even identify bacterial spores from vegetative bacteria. Some groups report differences (Shelly et al. 1980a, 1980b; Dalterio et al. 1987; Bronk and Reinisch 1991; Sorrell et al. 1994; Stephens 1998; Cheng et al. 1999; Seaver et al. 1999; Brosseau et al. 2000; Wichert et al. 2002; Agranovski et al. 2003; Sivaprakasam et al. 2004; Atkins et al. 2007; Thomas and Airola 2007), and others don't (Seaver et al. 1998; Cheng et al. 1999). This issue needs to be resolved prior to proceeding with developing the instrumentation and data interpretation algorithms that will be needed to optimize multiple-wavelength UV fluorescence/IR LIDAR detection of biological warfare agent aerosols.

Second, there is virtually no agreement between the fluorescence cross-sections measured for different species or even for identical species measured in different labs - the differences range up to two orders of magnitude (Seaver et al. 1998; Cheng et al. 1999; Sivaprakasam et al. 2004)! Measuring 
absolute fluorescence cross sections is difficult even with inanimate samples, and even more so when dealing with aerosol samples where the absolute concentration of biological material contained within the particle is not well known. But this experimental challenge cannot account for the two orders of magnitude differences seen. It has been noted, however, that the procedures used to prepare biological species strongly influence the fluorescence spectra and fluorescence quantum efficiencies - the growth medium, and the number of times the sample has been washed, are known to greatly influence fluorescence profiles; large differences in behavior have also been observed between wet and dry samples; and illumination laser intensities and local concentrations of different fluorophores contained within the biological sample also impact not only the measured fluorescence intensities, but also the wavelength-resolved spectral profiles (Farris et al. 1997; Fell et al. 1998; Johnson et al. 1999; Hill et al. 2001; Alimova et al. 2003; Kunnil et al. 2005; Sarasanandarajah et al. 2005b; Atkins et al. 2007).

Lastly, the simple observation of fluorescence after UV excitation is not a fail-safe way to distinguish a biological aerosol (even if it is not a warfare agent) from other ambient aerosols. It is well known that many organic chemicals, both naturally occurring (such as pinene) or anthropogenic (such as anthracene found in soot) fluoresce. Indeed Hill et al. (1999), Pan et al. (1999), and Halverson et al. (2003) report observing fluorescence from aerosols associated with tobacco smoke, chicken-house dust, gas and diesel soot, Arizona road dust, ammonium sulfate, and meadow oat dust; and others (Hargis et al. 1994) have shown that common volatile organic chemicals (VOCs, which combine to form urban aerosols) such as xylene, benzene, toluene, and acetone also have strong, broadband fluorescence between 300 and $600 \mathrm{~nm}$ after being excited with UV light ( $260 \mathrm{~nm})$.

\subsection{Multiple-Wavelength Elastic Backscatter LIDAR}

Originally this project was tasked to develop algorithms to optimize biological agent detection for a UV-LIF LIDAR system. However, it does not seem prudent to proceed with a detailed UV fluorescence analysis at this point given the large differences in the spectral profiles and cross sections that have been reported. After consultation with a number of experts who have fielded biological agent detectors, and listening to their concerns about the high rate of false positive detection that first- and second-generation two-wavelength fielded JBSDS prototypes have, it was decided that a better approach would be to look at the wavelength-dependent elastic backscatter profiles of a number of chemically distinct systems to see if data from this type of experiment can be used to categorize an approaching aerosol cloud (i.e., biological versus non-biological). This information could potentially augment whatever information can be gleaned from observing UV fluorescence. The inspiration for this research approach comes from a recent paper (Sindoni et al. 2006) that reported that two-dimensional (forward and backward) angular elastic scattering appears capable of resolving biological spores from spherical polystyrene particles. While multiplewavelength elastic backscatter LIDAR experiments cannot provide information on angular scattering patterns (which gives information about the internal structure of a particle and hence can differentiate between a layered spore and a solid organic sphere), the returns may provide some clues about the nature of an approaching aerosol cloud.

The amount of light elastically backscattered from an aerosol depends upon the size, shape and complex refractive index of the particles, and the number density. The scattering intensities are also strongly affected by the wavelength of the incident light. Biological warfare agents may have one, perhaps two, physical properties (i.e., size or shape) in common with ambient aerosols, but the 
combination of optical and physical characteristics, as well as the wavelength response, should be distinctive enough to provide a characteristic signature.

Sporulated biological warfare agents are generally rod-shaped, whereas ambient background aerosols (such as sulfates or hydrophilic organics) are generally spherical, because the surface tension of water in the latter systems keeps them this way. Biological warfare agent aerosols typically consist of single rodshaped spores with a spherical equivalent radius about 0.63 microns. It is noted here that the number of spores contained within an aerosol will depend upon the method of dispersal, but to be effective an enemy would design a weapon system to release single spores because clumping inhibits wide-area dispersion (i.e., heavy particles will not remain airborne for long). Biological warfare agent aerosols will also not be affected by humidity because their water vapor uptake kinetics are slow to begin with, and the hydrophobic surfactants that are used to prevent clumping further elongate the response time.

Obviously there can be many different types of ambient aerosols. Considering that most biological sensors will be tested at the Edgewood Chemical and Biological Center, this work was restricted to comparing backscattering results from biological agents with aerosols associated with a semi-clean urban environment - specifically sulfate, volatile organic chemical (VOC), soot, and silicate (road dust) particles. These aerosols are much smaller than biological agent ones - their average radii range between 0.1 and 0.3 microns. Sulfate and VOC aerosols will respond to changes in local weather conditions; specifically, they will grow in size with increasing humidity, and this is addressed in this report. The soot and silicate aerosols are unaffected by humidity because they are hydrophobic.

Biological aerosols' optical properties are also different than those found for ambient aerosols. Tuminello et al. and Arakaw et al. have measured the wavelength dependence of the real (n) and imaginary (k) parts of the complex refractive index of Bacillus subtilis var. niger spores (also known as Bacillus globigii) and Erwinia herbicola which is a vegetative bacterium (Tuminello et al. 1997; Arakawa et al. 2003). These values are shown in Figure 1.1, along with values that have been measured for sulfate, water, VOCs, Arizona road dust, and soot (Hale and Querry 1973; Toon et al. 1976; Querry 1987; Hess et al. 1998; Marley et al. 2001).

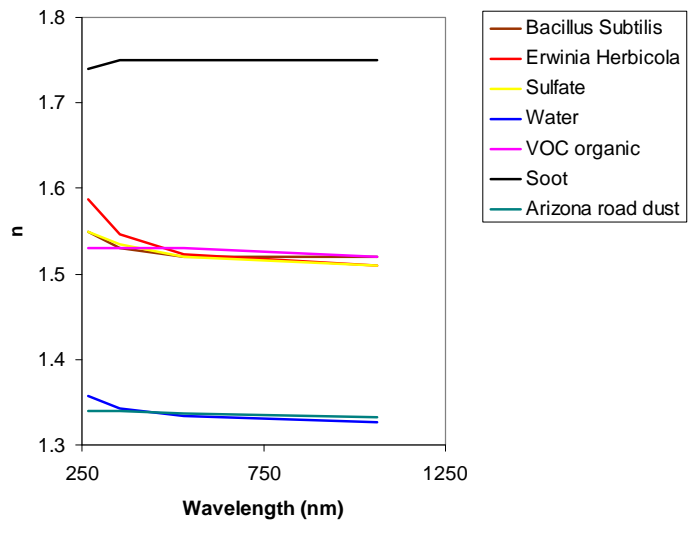

(a)

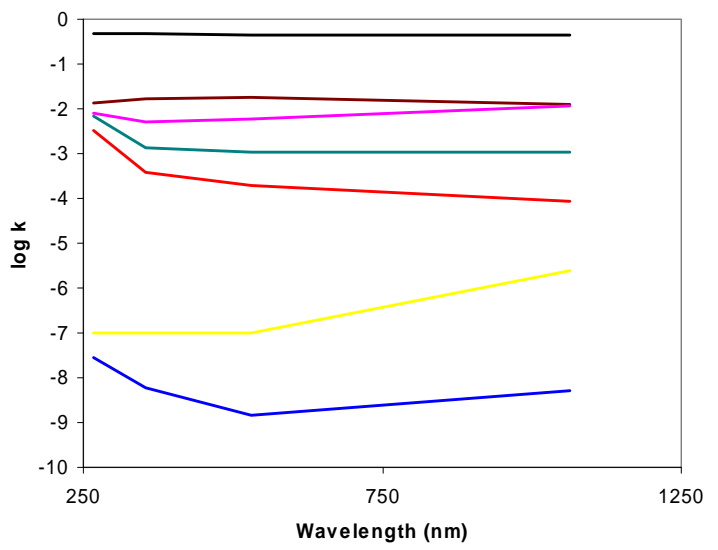

(b)

Figure 1.1. Measured Wavelength Dependence of (a) Real and (b) Imaginary Parts of the Refractive Index of Various Aerosol Components 
Given that biological warfare agent aerosol size, shape, and wavelength-dependent optical properties are different than those associated with ambient aerosols, it is not difficult to imagine that it may be possible to use multiple-wavelength elastic backscatter measurements to distinguish harmful clouds from innocuous ones. In the following, Mie scattering theory is used to calculate elastic backscattering cross sections for two biological systems - Bacillus Subtilis var. niger spores (an anthrax simulant) and Erwinia Herbicola (a vegetative bacteria) - and four ambient aerosol systems - sulfate, VOC-based, soot and Arizona road dust. Elastic-backscatter cross sections are calculated at four wavelengths - 266, 355, 532, and $1064 \mathrm{~nm}$ - which can be generated from a single Nd:YAG laser system. The cross sections and the relative wavelength response are compared to see if the signature from biological systems is sufficiently distinctive to enable categorization of aerosol type. 


\subsection{LIDAR Equation Modeling}

The LIDAR equation model used is a straightforward implementation of standard models for elastic backscattering LIDAR, where the scattering target is assumed to be volume scattering from atmospheric molecules and aerosols (Measures 1984; Warren et al. 2004).

$$
P_{r}(R)=E \frac{c}{2} \tau_{\text {optics }} f_{\text {over }} \tau_{\text {atm }}^{2} \frac{A}{R^{2}}\left(\frac{\beta_{\text {ambient }}}{(8 \pi / 3)}+\frac{\sigma_{b}}{4 \pi} N_{b i o}(R)\right)(W)
$$

where:

$$
\begin{aligned}
P_{r}(R) & =\text { received laser power }(\mathrm{W}) \\
E & =\text { laser energy per pulse }(\mathrm{J}) \\
c & =\text { speed of light }(\mathrm{m} / \mathrm{s}) \\
\tau_{\text {optics }} & =\text { geometrical efficiency of transmit/receive paths (unitless) } \\
f_{\text {over }} & =\text { geometrical overlap function (unitless) } \\
\tau_{\text {atm }} & =\text { atmospheric transmission (unitless) } \\
A & =\text { telescope area }\left(\mathrm{m}^{2}\right) \\
R & =\text { range }(\mathrm{m}) \\
\beta_{\text {ambient }} & =\text { ambient volume scattering coefficient }\left(\mathrm{m}^{-1}\right) \\
\sigma_{b} & =\text { backscattering cross section of single aerosol particle }\left(\mathrm{m}^{2}\right) \\
N_{\text {bio }}(R) & =\text { concentration of bio-aerosol particles }\left(\mathrm{m}^{-3}\right)
\end{aligned}
$$

In Eq. (2.1), the scattering aerosol is separated into two components. The ambient component characterized by $\beta_{\text {ambient }}$ and the component due to a discrete plume of bio-aerosol particles characterized by number density $N_{b i o}$ and backscattering cross-section $\sigma_{b}$. 
2.2 


\subsection{Aerosol Modeling for Biological Particles}

The aerosol modeling introduced in the previous section has two major components - the background aerosols and the bio-particle aerosols. The ambient aerosols can be further separated into the molecular, or Rayleigh, scattering component and the particulate scattering component.

\subsection{Ambient Aerosol Modeling}

The total ambient aerosol volumetric scattering coefficient is the sum of the naturally occurring aerosol contribution, characterized by $\beta_{\text {aero }}$, and the molecular/Rayleigh contribution, characterized by $\beta_{\text {rayl }}$,

$$
\beta_{\text {ambient }}=\beta_{\text {aero }}+\beta_{\text {rayl }}\left(\mathrm{m}^{-1}\right)
$$

where:

$$
\begin{aligned}
\beta_{\text {ambient }} & =\text { volume scattering coefficient due to ambient aerosols and molecules }\left(\mathrm{m}^{-1)}\right. \\
\beta_{\text {aero }} & =\text { volume scattering coefficient due to natural aerosols }\left(\mathrm{m}^{-1}\right) \\
\beta_{\text {rayl }} & =\text { volume scattering coefficient due to molecular Rayleigh scattering }\left(\mathrm{m}^{-1}\right)
\end{aligned}
$$

\subsubsection{Rayleigh Molecular Scattering Model}

The Rayleigh scattering model is derived in detail by Bucholtz (1995), and the results are summarized here for completeness and for notational uniformity with the LIDAR model detailed in Section 2. The Rayleigh scattering cross-section is given by

$$
\sigma(\lambda)=\frac{24 \pi^{3}}{\lambda^{4} N_{s}^{2}} \frac{\left(n_{s}^{2}-1\right)^{2}}{\left(n_{s}^{2}+2\right)^{2}} \frac{6+3 \rho_{n}}{6-7 \rho_{n}}\left(\mathrm{~cm}^{2}\right)
$$

where:

$$
\begin{aligned}
\sigma(\lambda) & =\text { scattering cross section }\left(\mathrm{cm}^{2}\right) \\
\lambda & =\text { wavelength }(\mathrm{cm}) \\
n_{S} & =\text { refractive index for standard air at } \lambda \text { (unitless) } \\
N_{S} & =\text { molecular number density for standard air }=2.54743 \times 10^{19}\left(\mathrm{~cm}^{3}\right) \\
\rho_{n} & =\text { depolarization factor at } \lambda \text { (unitless) }
\end{aligned}
$$

The total volume scattering coefficient is given by

$$
\beta_{s}(\lambda)=N_{s} \sigma(\lambda) \cdot 100\left(\mathrm{~m}^{-1}\right)
$$


where:

$$
\begin{aligned}
\beta_{S}(\lambda) & =\text { Rayleigh volume scattering coefficient (standard air) }\left(\mathrm{m}^{-1}\right) \\
N_{S} & =\text { molecular number density for standard air }=2.54743 \times 10^{19}\left(\mathrm{~cm}^{3}\right) \\
\sigma(\lambda) & =\text { scattering cross section }\left(\mathrm{cm}^{2}\right) \\
\lambda & =\text { wavelength }(\mathrm{cm}) \\
100 & =\text { factor to convert from } \mathrm{cm}^{-1} \text { to } \mathrm{m}^{-1}
\end{aligned}
$$

Bucholtz used empirical formulas for $n_{S}$ and tabulated results for $\rho_{n}$ and derived an approximate analytical formula for $\sigma(\lambda)$ using a least squares fit to the results to obtain,

$$
\sigma(\lambda)=A \lambda^{-(B+C \lambda+D / \lambda)}\left(\mathrm{cm}^{2}\right)
$$

where:

$$
\begin{aligned}
& \sigma(\lambda)=\text { scattering cross-section }\left(\mathrm{cm}^{2}\right) \\
& \lambda=\text { wavelength }(\mu \mathrm{m}) \\
& A=3.01577 \times 10^{-28} \\
& B=3.55212 \\
& C=1.35579 \\
& 0.2 \leq \lambda \leq 0.5 \mu \mathrm{m} \\
& D=0.11563 \\
& A=4.01061 \times 10^{-28} \\
& B=3.99668 \\
& C=1.10298 \times 10^{-3} \\
& \lambda>0.5 \mu \mathrm{m} \\
& D=2.71393 \times 10^{-2}
\end{aligned}
$$

The volume scattering coefficient, $\beta$, scales with number density, so at any temperature or pressure,

$$
\beta_{\text {rayl }}(\lambda)=\beta_{s} \frac{N}{N_{s}}=\beta_{s} \frac{P}{P_{s}} \frac{T_{s}}{T}\left(\mathrm{~m}^{-1}\right)
$$

where:

$$
\begin{aligned}
\beta_{\text {rayl }}(\lambda) & =\text { Rayleigh volume scattering coefficient }\left(\mathrm{m}^{-1}\right) \\
\beta_{S} & =\text { Rayleigh volume scattering coefficient }(\text { standard air })\left(\mathrm{m}^{-1}\right) \\
N & =\text { molecular number density }\left(\mathrm{cm}^{3}\right) \\
N_{S} & =\text { molecular number density for standard air }=2.54743 \times 10^{19}\left(\mathrm{~cm}^{3}\right) \\
P & =\text { atmospheric pressure (bars) } \\
P_{S} & =\text { standard atmospheric pressure }=1013.25 \text { mbars (bars) } \\
T & =\text { temperature }(\mathrm{K}) \\
T_{S} & =\text { standard air temperature }=288.15 \mathrm{~K}(\mathrm{~K})
\end{aligned}
$$


The angular dependence of the scattering is defined by

$$
\beta(\theta, \lambda)=\frac{\beta_{\text {rayl }}(\lambda)}{4 \pi} P_{r a y}(\theta)\left(\mathrm{m}^{-1}\right)
$$

where:

$$
\begin{aligned}
& \beta(\theta, \lambda)=\text { angular volume scattering coefficient }\left(\mathrm{m}^{-1}\right) \\
& P_{r a y}(\theta)=\frac{3}{4}\left(1+\cos ^{2} \theta\right)=\text { Rayleigh phase function (unitless) }
\end{aligned}
$$

For backscatter, $\theta=\pi$, and $\mathrm{P}_{\text {ray }}=3 / 2$. Kong (1986, p. 482-485) has $\sigma_{b}=3 \sigma_{s} / 2$ for scattering from a small dielectric sphere where $\sigma_{b}$ is the backscatter cross-section (equivalent to RCS), and $\sigma_{S}$ is the total scattering cross section. Note that $3 / 2$ is also the directivity of a Hertzian dipole, which makes sense for scattering from infinitesimal spheres.

$$
\beta_{\text {back }}(\lambda)=\frac{N \sigma(\lambda) \cdot 100}{4 \pi} \frac{3}{2}=\frac{\beta_{\text {rayl }}(\lambda)}{4 \pi} \frac{3}{2}\left(\mathrm{~m}^{-1}\right)
$$

where:

$$
\beta_{\text {back }}(\lambda)=\text { volume backscattering coefficient due to mol Rayleigh scattering }\left(\mathrm{m}^{-1}\right)
$$

Note that this includes the $4 \pi$ term; take care not to duplicate.

\subsubsection{Natural Aerosol Scattering Model}

The atmospheric attenuation due to Mie scattering from naturally occurring atmospheric aerosols is given by Measures (1984, p. 143) as

$$
\beta_{M}=\frac{3.91}{R_{v}}\left(\frac{0.55}{\lambda}\right)^{q} \quad\left(\mathrm{~km}^{-1}\right)
$$

where:

$$
\begin{aligned}
\beta_{M} & =\text { attenuation coeff. }\left(\mathrm{km}^{-1}\right) \\
R_{v} & =\text { visibility }(\mathrm{km}) \\
q & = \begin{cases}0.585 R_{v}^{1 / 3} \quad\left(R_{v} \leq 6 \mathrm{~km}\right) \\
1.3 \quad \text { (avg condictions) } \\
2 \quad \text { (clear conditions) }\end{cases} \\
\lambda & =\text { wavelength }(\mu \mathrm{m})
\end{aligned}
$$


Warren et al. (2004) use this formula for the total volume scattering coefficient and makes an adjustment to remove the Rayleigh scattering (at $0.55 \mu \mathrm{m}$ ) which is assumed to be included in this empirical formula. The factors of 0.001 and 1000 are used to convert $\mathrm{km}^{-1}$ to $\mathrm{m}^{-1}$ or m$^{-1}$ to $\mathrm{km}^{-1}$, respectively.

$$
\beta_{\text {aero }}=0.001\left(\frac{3.91}{R_{v}}-1000 \beta_{\text {rayl }}(0.55)\right)\left(\frac{0.55}{\lambda}\right)^{q}\left(\mathrm{~m}^{-1}\right)
$$

where

$$
\begin{aligned}
\beta_{\text {aero }} & =\text { vol scattering coeff. for natural aerosols }\left(\mathrm{m}^{-1}\right) \\
\beta_{\text {rayl }} & =\text { Rayleigh vol scattering coeff. for natural aerosols }\left(\mathrm{m}^{-1}\right) \\
R_{v} & =\text { visibility }(\mathrm{km}) \\
q & = \begin{cases}0.585 R_{v}^{1 / 3} \quad\left(R_{v} \leq 6 \mathrm{~km}\right) \\
1.3 & \text { (avg condictions) } \\
2 & \text { (clear conditions) }\end{cases} \\
\lambda & =\text { wavelength }(\mu \mathrm{m})
\end{aligned}
$$

\subsection{Aerosol Mie Scattering}

Bio-particles will typically have sizes comparable to or larger than the wavelength of the elastic scattering LIDAR system used to detect them. For spherical particles, Mie scattering models have been developed that can be used to compute the optical properties of individual spherical particles and particlesize distributions (Bohren and Huffman 1983; Mishchenko et al. 2002).

Mie scattering calculations characterize the scattered and absorbed optical power due to a homogeneous, isolated spherical particle illuminated by an optical plane wave with power density $I_{i}\left(\mathrm{~W} / \mathrm{m}^{2}\right)$, as depicted in Figure 3.1. The particle is characterized by radius $a$, and complex index of refraction $m$. Upon striking the particle, optical power is either scattered by reflection from the particle, absorbed within the particle, or passes around the particle. The scattered power is $W_{\text {sca }}$ and the absorbed power is $W_{a b s}$. The extinction is the combined energy loss due to both absorption and scattering,

$$
W_{e x t}=W_{s c a}+W_{a b s}
$$

Extinction, scattering, and absorption are characterized by cross-sections, which are defined by 


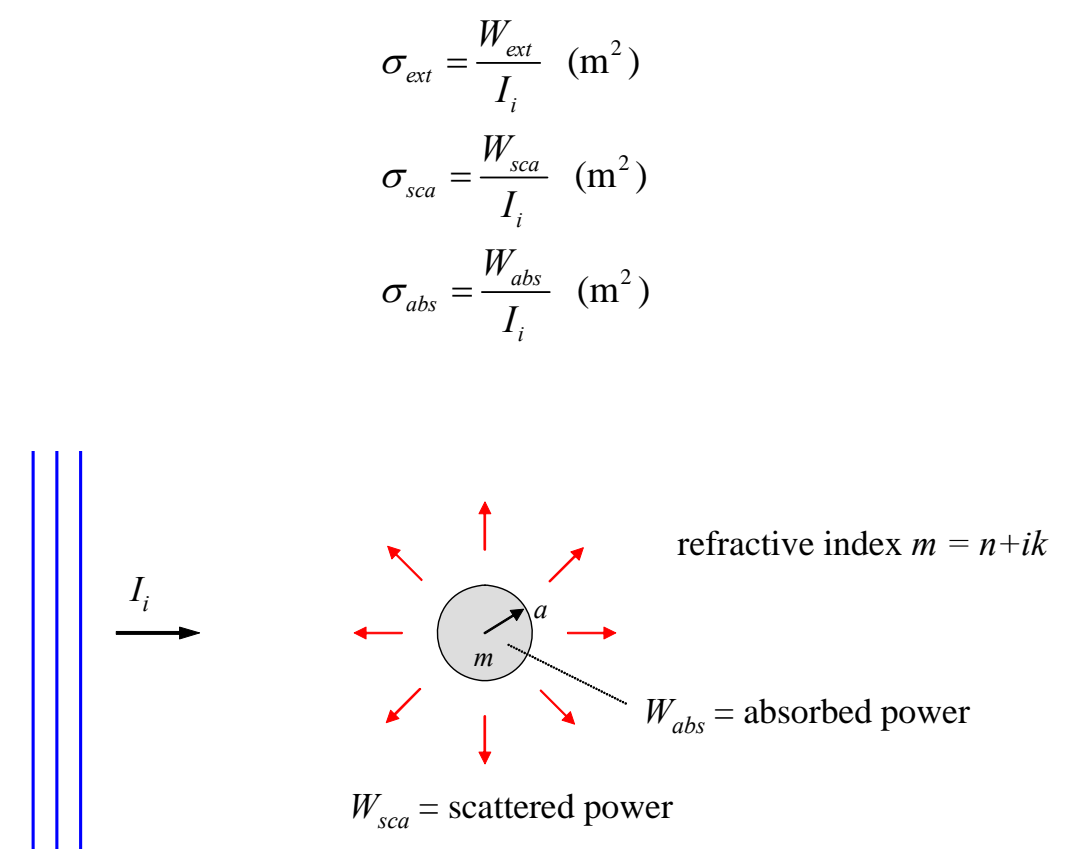

Figure 3.1. Spherical Dielectric Particle Illuminated by a Plane Optical Wave

Mie efficiencies are cross sections normalized by the particle cross-sectional area $\left(\pi a^{2}\right)$ and are given by

$$
\begin{aligned}
& Q_{e x t}=\frac{\sigma_{e x t}}{\pi a^{2}}\left(\mathrm{~m}^{2}\right) \\
& Q_{s c a}=\frac{\sigma_{s c a}}{\pi a^{2}}\left(\mathrm{~m}^{2}\right) \\
& Q_{a b s}=\frac{\sigma_{a b s}}{\pi a^{2}}\left(\mathrm{~m}^{2}\right)
\end{aligned}
$$

A backscattering cross section is similar to the scattering cross section, except that the direction of scattering is limited to the backscattering direction rather than averaged over all directions. This cross section is particularly relevant to the LIDAR system since the return optical power from the particle is proportional to the backscattering cross section $\sigma_{b}$, with corresponding Mie efficiency given by

$$
Q_{b}=\frac{\sigma_{b}}{\pi a^{2}}
$$

Mie scattering derivations (given in Bohren and Huffman [1983]) expand the internal and external electromagnetic fields as superpositions of spherical Bessel functions with modal amplitudes $a_{n}, b_{n}, c_{n}$, and $d_{n}$, where $n$ indicates the mode number. Matching boundary conditions at the surface of the sphere allows for solution to the $a_{n}, b_{n}, c_{n}$, and $d_{n}$, values. The Mie efficiencies (and therefore cross sections) are then determined from the values of the $a_{n}$ 's and $b_{n}$ 's as 


$$
\begin{aligned}
& Q_{\text {sca }}=\frac{2}{x^{2}} \sum_{n=1}^{\infty}(2 n+1)\left(\left|a_{n}\right|^{2}+\left|b_{n}\right|^{2}\right) \\
& Q_{e x t}=\frac{2}{x^{2}} \sum_{n=1}^{\infty}(2 n+1) \operatorname{Re}\left(a_{n}+b_{n}\right) \\
& Q_{a b s}=Q_{e x t}-Q_{s c a} \\
& Q_{b}=\frac{1}{x^{2}}\left|\sum_{n=1}^{\infty}(2 n+1)(-1)^{n}\left(a_{n}-b_{n}\right)\right|^{2}
\end{aligned}
$$

Cross sections are obtained by multiplying the efficiencies in Eq. (3.14) by the particle's cross sectional area $\pi a^{2}$. The total number of modes theoretically extends to infinity, but can be truncated to a reasonable and finite value that depends on the size of the particle relative to the optical wavelength. Most Mie scattering calculations compute the cross sections as a function of the size parameter $x$, which is essentially a normalized radius and is given by

$$
x=k a=\frac{2 \pi}{\lambda} a
$$

where $k=2 \pi / \lambda$ is the wavenumber as normally defined in electromagnetics texts.

Figure 3.2 through Figure 3.4 show the Mie efficiencies for low-loss ( $m=1.4+0.005 i)$, medium-loss $(m=1.4+0.05 i)$, and high-loss $(m=1.4+0.5 i)$ cases. Note the oscillatory nature of the backscatter, extinction, and scattering efficiencies. This is due to optical resonances within the spherical particle. For the medium- and high-loss cases, the optical resonances are damped more highly and the efficiencies converge to near-constant values for larger values of the size parameter.

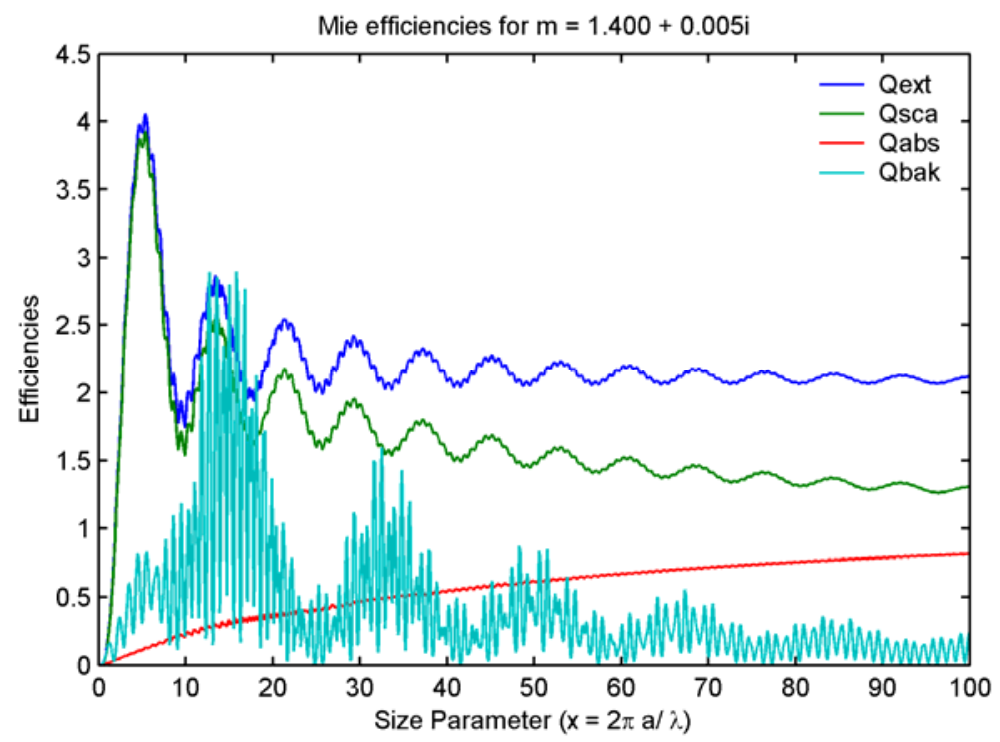

Figure 3.2. Mie Efficiencies for a Low-Loss Case with $m=1.4+0.005 i$ 


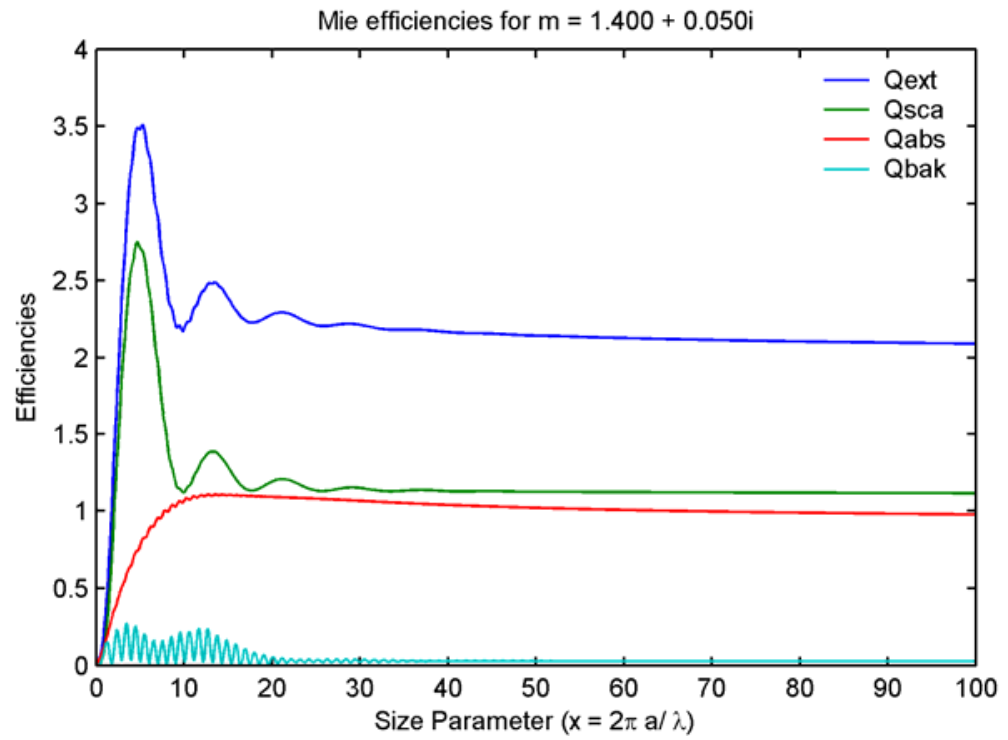

Figure 3.3. Mie Efficiencies for a Medium-Loss Case with $m=1.4+0.05 i$

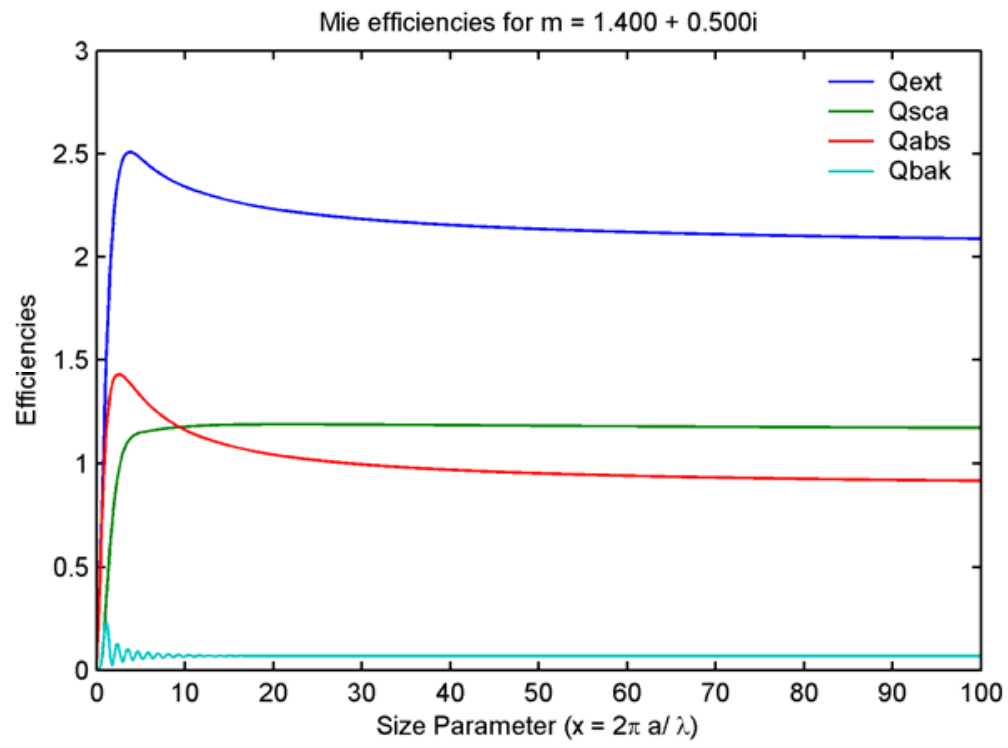

Figure 3.4. Mie Efficiencies for a High-Loss Case with $m=1.4+0.5 i$

Limiting cases in which the particles are assumed to be either very small or very large can be used to help verify the accuracy of the Mie efficiency/cross-section calculations. For small particles $(k a<<1)$ the backscattering efficiency is expected to be (Kong 1986),

$$
Q_{b} \cong 4(k a)^{4}\left|\frac{m^{2}-1}{m^{2}+2}\right|^{2} \quad(k a<<1)
$$


This is plotted along with the Mie calculation in Figure 3.5. Excellent agreement is observed when $k a<<1$, as is expected. Large particles ( $k a>>1)$ with sufficient loss are expected to have backscattering efficiencies that are approximately equal to the Fresnel power reflection coefficient and are given by (Bohren and Huffman 1983),

$$
Q_{b} \cong\left|\frac{1-m}{1+m}\right|^{2} \quad(k a>>1)
$$

This result assumes that the loss (imaginary component of the complex dielectric constant) is sufficiently large so that any optical energy that enters the particle is absorbed without re-radiating. In the large sphere limit, the extinction efficiency is expected to be (Bohren and Huffman 1983)

$$
Q_{e x t} \cong 2 \quad(k a>>1)
$$

Figure 3.6 shows the Mie backscattering efficiency plotted with the large sphere limiting function value given by Eq. (3.17). Agreement is excellent for size parameters greater than approximately 50 . Figure 3.7 shows the Mie extinction efficiency plotted with the large sphere limiting function given by Eq. (3.18). Agreement is apparent in this case only for very large spheres with size parameter exceeding 100.

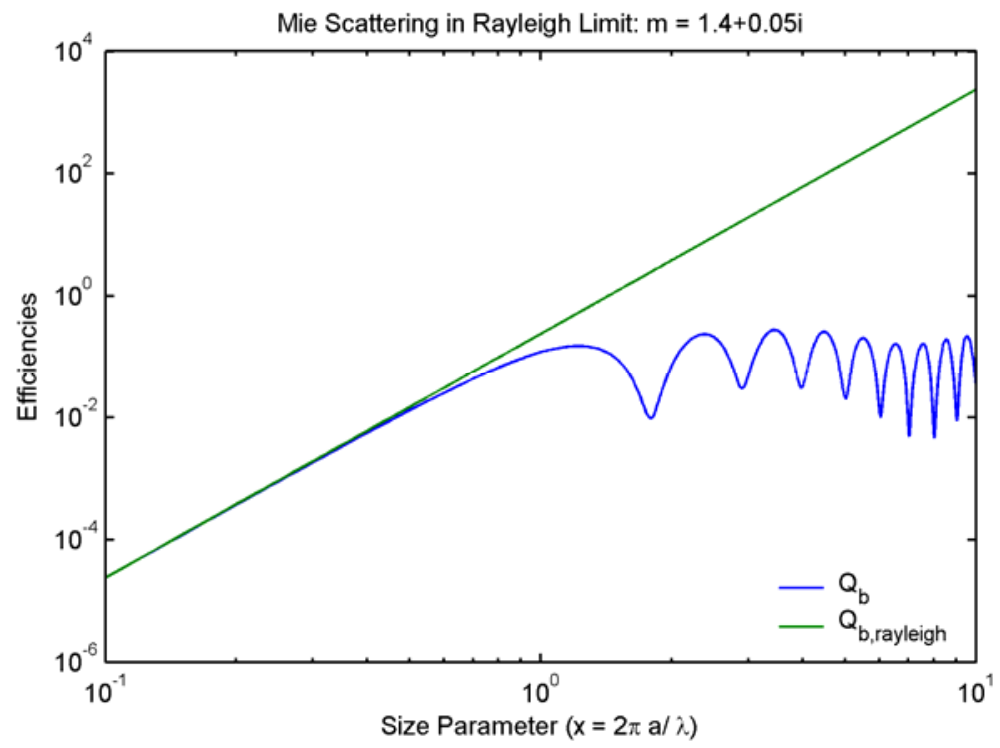

Figure 3.5. Mie Backscattering Efficiency in the Small Sphere (Rayleigh) Limit 


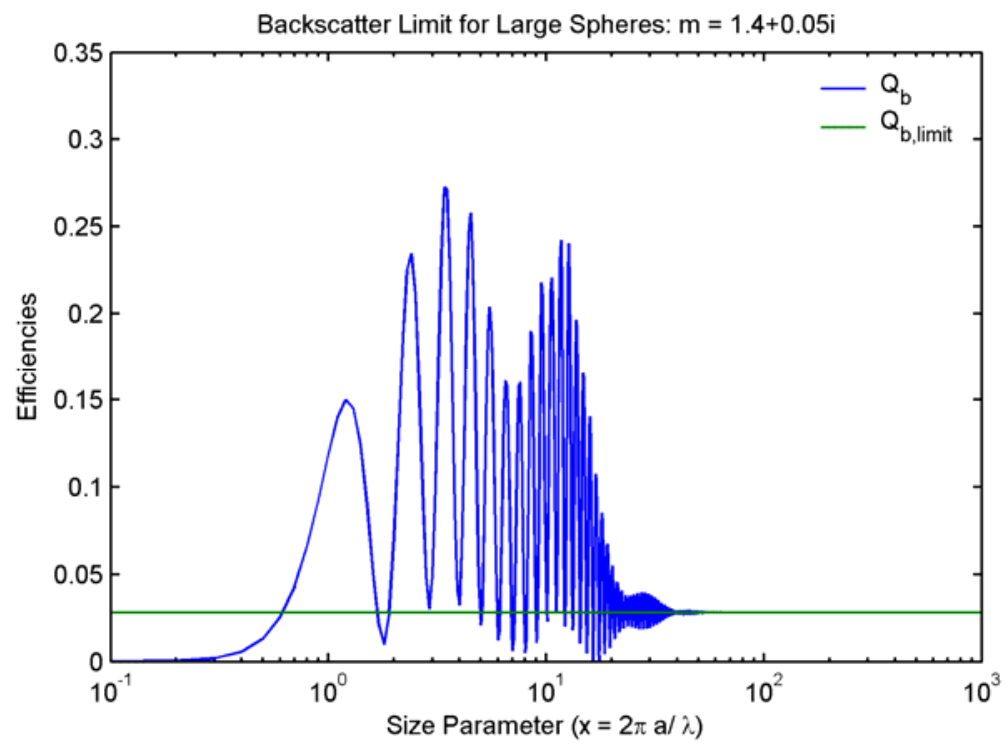

Figure 3.6. Mie Backscattering Efficiency in the Large Sphere Limit

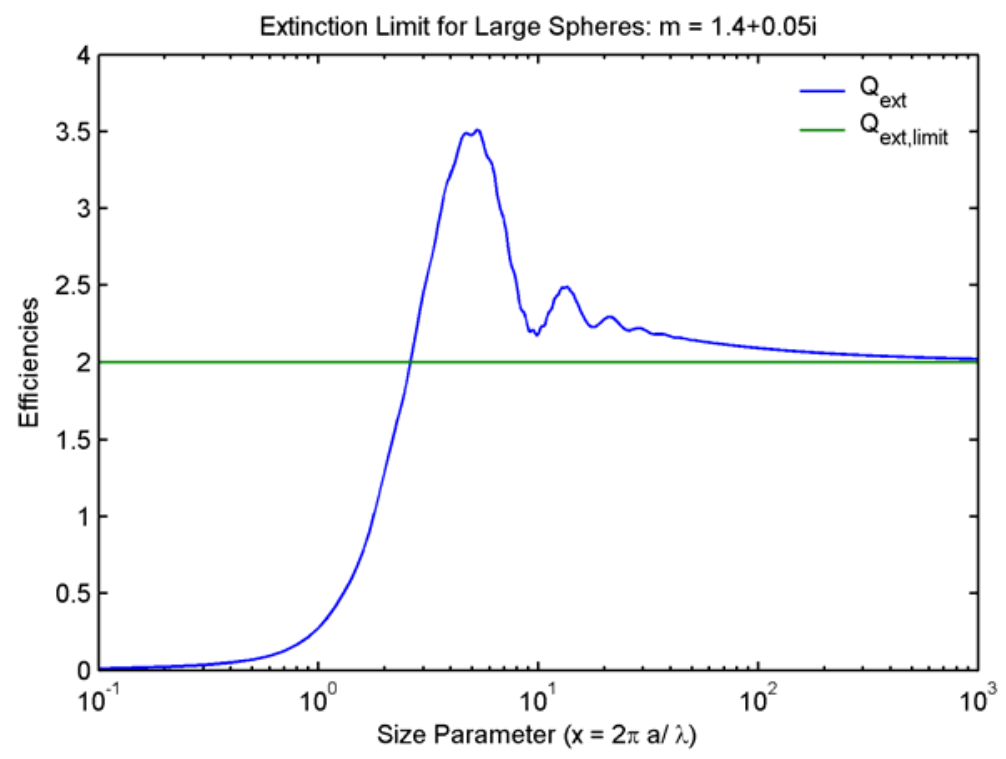

Figure 3.7. Mie Extinction Efficiency in the Large Sphere Limit

The preceding analysis was for individual spherical dielectric particles. Aerosols are modeled as polydisperse distributions of spherical dielectric particles. Assuming that the particle locations are sufficiently random, the effect of the size distribution can be reduced to a weighted average of cross sections over the size distribution. Following Mishchenko et al. (2002), several different particle size distribution functions have been implemented in the Bio-Spectral Algorithm Stimulator (BSAS). These include the log-normal, modified gamma, power law, gamma, modified power law, and modified binomial log-normal distribution functions. 
Log-normal distribution

$$
n(r)=\text { constant } \times r^{-1} \exp \left(-\frac{\left(\ln r-\ln r_{g}\right)^{2}}{2 \ln ^{2} \sigma_{g}}\right)
$$

Modified gamma distribution

$$
n(r)=\text { constant } \times r^{\alpha} \exp \left(-\frac{\alpha r^{\gamma}}{\gamma r_{c}^{\gamma}}\right)
$$

Power law distribution

$$
n(r)= \begin{cases}\text { constant } \times r^{-3}, & r_{1} \leq r \leq r_{2} \\ 0 & \text { otherwise }\end{cases}
$$

Gamma distribution

$$
n(r)=\text { constant } \times r^{(1-3 b) / b} \exp \left(-\frac{r}{a b}\right), \quad b \in(0,0.5)
$$

Modified power law distribution

$$
n(r)=\left\{\begin{array}{c}
\text { constant, } \quad r<r_{1} \\
\text { constant } \times\left(r / r_{1}\right)^{\alpha}, \quad r_{1} \leq r \leq r \\
0, \quad r>r_{2}
\end{array}\right.
$$

Modified bimodal log-normal distribution

$$
n(r)=\text { constant } \times r^{-4}\left\{\exp \left(-\frac{\left(\ln r-\ln r_{g 1}\right)^{2}}{2 \ln ^{2} \sigma_{g 1}}\right)+\gamma \exp \left(-\frac{\left(\ln r-\ln r_{g 2}\right)^{2}}{2 \ln ^{2} \sigma_{g 2}}\right)\right\}
$$

where the constant is determined so that the integral of $n(r)$ from zero to infinity is unity.

The log-normal distribution is typically used by the BSAS for aerosol calculations. In the log-normal distribution function (Eq. 3.19), the factor $r_{g}$ can be considered to be one-half of the count median diameter (CMD). Note that this is not the maximum (mode) of the distribution function. The HatchChoate equations define the relationship between the CMD and other diameters of interest. These include the mass median diameter (MMD), surface median diameter (SMD), diameter of average volume $\left(d_{v}\right)$, and diameter of average surface $\left(d_{s}\right)$. These relationships are defined as: 


$$
\begin{aligned}
\mathrm{CMD} & =2 r_{g} \\
\mathrm{MMD} & =\mathrm{CMD} \times \exp \left(3 \ln ^{2} \sigma_{g}\right) \\
\mathrm{SMD} & =\mathrm{CMD} \times \exp \left(2 \ln ^{2} \sigma_{g}\right) \\
d_{V} & =\mathrm{CMD} \times \exp \left(1.5 \ln ^{2} \sigma_{g}\right) \\
d_{S} & =\mathrm{CMD} \times \exp \left(1.0 \ln ^{2} \sigma_{g}\right)
\end{aligned}
$$

Log-normal distribution in the BSAS aerosol calculations are usually specified by their MMD and $\sigma_{g}$ values.

The effective aerosol cross sections are calculated simply as a weighted average (integral) over the particle size distribution as,

$$
\langle\sigma\rangle=\int_{r_{\min }}^{r_{\max }} \sigma(r) n(r) d r
$$

where $\sigma$ represents $\sigma_{s c a}, \sigma_{a b s}, \sigma_{e x t}$, or $\sigma_{b}$.

This integration is conveniently and accurately performed using sub-divided Gauss-Legendre quadrature (Press et al. 1992). Over a single sub-interval from $X_{1}$ to $X_{2}, N^{\text {th }}$ order Gauss-Legendre quadrature calculates weights $w_{i}$ and positions $x_{i}$ so that integration is exact for polynomials up to order $N$. For a general function $f(x)$,

$$
\int_{X_{1}}^{X_{2}} f(x) d x \cong \sum_{i=1}^{N} w_{i} f\left(x_{i}\right)
$$

Additional accuracy is obtained by applying the Gauss-Legendre quadrature over many sub-intervals. Multiplication by the aerosol distribution's number density (particles $/ \mathrm{m}^{3}$ ) scales the overall scattering, absorption, extinction, and backscatter terms.

To verify proper implementation of the distribution functions and effective, or ensemble average, cross-section calculations, the following aerosol distribution was analyzed (Mishchenko et al. 2002). A power law distribution was selected with the following parameters,

$$
\begin{aligned}
\lambda & =0.65 \mu \mathrm{m} \\
m & =1.53+0.008 i \\
r_{\text {min }} & =0.245830 \mu \mathrm{m} \\
r_{\max } & =1.194170 \mu \mathrm{m}
\end{aligned}
$$

with the following results 


$$
\begin{aligned}
\left\langle\sigma_{\text {ext }}\right\rangle & =1.92604 \mu \mathrm{m}^{2} \text { (Mischenko) } \\
& =1.9260 \mu \mathrm{m}^{2} \text { (BSAS) } \\
\left\langle\sigma_{\text {sca }}\right\rangle & =1.78033 \mu \mathrm{m}^{2} \text { (Mischenko) } \\
& =1.7803 \mu \mathrm{m}^{2} \text { (BSAS) }
\end{aligned}
$$

These results are in near-perfect agreement providing strong evidence that the integration over the distribution function is implemented properly.

\subsection{T-matrix Scattering}

Many bio-particles are significantly non-spherical, and cannot accurately be approximated as spherical. In this case, an alternative to the Mie scattering calculation technique is required. The T-matrix method is a numerical technique for solving Maxwell's equations to provide accurate scattering cross sections for non-spherical particles (Mishchenko et al. 2002). In practice, the technique is limited to particles that can be modeled as bodies of revolution. In particular, prolate and oblate spheroidal particles are conveniently modeled using this technique. Versatile FORTRAN code that implements this technique is available at http://www.giss.nasa.gov/ crmim/ , and this code was compiled and used to perform backscatter cross-section estimates for modeling non-spherical bio-particles. 


\subsection{Aerosol Optical and Physical Properties}

Because most biosensor tests take place at the Edgewood Chemical and Biological Center, it was decided to model the release of a cloud of biological warfare agents in a relatively clean urban environment. In this case, sulfate aerosols will be the most prevalent species, but varying amounts of hygroscopic aerosols that contain volatile organic chemicals, soot aerosols, and road dust aerosols may also be seen. In the following paragraphs, the physical and optical properties of the different systems that were used for input into the Mie/T-matrix calculations are described.

\subsection{Ambient Sulfate Aerosols}

It was decided that the cloud condensation nuclei (CCN) are comprised of dry ammonium sulfate and will determine backscatter intensities for a relative humidity that varies between 35\% and 95\% (these boundaries eliminate problems that come from phase changes or abrupt growth change due to deliquescence). Both dry and wet aerosols were assumed to be spherical. For the modeling, the following was adopted:

There is a single-mode log-normal distribution of "seed" aerosol sizes, also known as dry aerosols or CCN. The single-mode distribution has the following form

$$
n\left(r_{d r y}\right)=d N / d r=\frac{N_{o} \exp \frac{-\ln r_{d r y}-\ln r_{o}}{2(\ln \sigma)^{2}}}{r \ln \sigma(2 \pi)^{1 / 2}}
$$

where $r_{\text {dry }}$ is the aerosol particle radius, $N_{o}$ is the total number density, $r_{0}$ is the geometric mean radius of the dry aerosol distribution, and $\sigma$ is the geometric standard deviation. For the purposes of building a model that can be varied with ease, it would be reasonable to have $\mathrm{N}_{\mathrm{o}}, \mathrm{r}_{\mathrm{o}}$ and $\sigma$ as input variables.

For the initial modeling studies, the dry aerosol distribution was assumed to have

$$
\begin{aligned}
\mathrm{N}_{\mathrm{o}} & =8.3 \times 10^{6} \text { particles } / \mathrm{m}^{3} \\
\mathrm{r}_{\mathrm{o}} & =0.1355 \times 10^{-6} \mathrm{~m} \\
\sigma & =1.5
\end{aligned}
$$

based on the values quoted by others (Taylor and Penner 1994). When seed aerosols are exposed to humid air they can accumulate water and grow. The growth depends strongly on the chemical composition of the original seed particle. Several different aerosol types have been studied in the laboratory and in the field and have been modeled theoretically. Sulfate aerosols have been studied the most because these are the most prevalent species in the troposphere and stratosphere.

To describe the growth of a sulfate aerosol in a humid environment a growth factor, $\eta$, was defined as the ratio of the aerosol particle radius $\mathrm{r}$ at a specified humidity, $\mathrm{H}$, (the relative humidity normalized to 1 ) to the radius of the corresponding dry aerosol, $r_{d r y}$. 


$$
r / r_{d r y}=\eta\left(r_{d r y}, H\right)
$$

The wet size particle distribution $n(r)$ is related to the dry size distribution $n\left(r_{\text {dry }}\right)$ through

$$
n\left(r_{d r y}\right) \geq n(r)=\frac{n\left(\frac{r}{\eta}\right) d\left(\frac{r}{\eta}\right)}{d r}
$$

In exact theory, $\eta$ is sensitive to $r_{d r y}$, and to get an exact value for $\eta$ can involve a lot of integration and iteration, activity coefficients, etc. However, there are physical ranges where the exact function can be replaced with an empirical one ( $\mathrm{Li}$ et al. 2001) — specifically, for dry aerosol radii between 0.1 and $1 \mu \mathrm{m}$ and relative humidity between 5 and 95\% — and introduce, at most, a 5\% error. Because the boundary humidity range and aerosol size distribution are in the range of what is being modeled, the following empirical equation can be used;

$$
\eta\left(r_{d}, H\right)=\exp \left[\frac{l_{1}+l_{2} H+l_{3}}{\left(H-l_{4}\right)^{2}}\right]
$$

where for ammonium sulfate aerosols

$$
\begin{aligned}
& l_{1}=-0.08082 \\
& l_{2}=0.5121 \\
& l_{3}=0.004823 \\
& l_{4}=1.070 .
\end{aligned}
$$

Equation (4.4) and the initial dry aerosol distribution parameters can then be used to determine the wet aerosol size distribution as a function of humidity. Again Eq. (4.1) is used with $\mathrm{N}_{\mathrm{o}}$ and $\sigma$ the same as used to describe the dry aerosol distribution and now $\mathrm{r}_{\mathrm{o} \text {,wet }}=\mathrm{r}_{\mathrm{o} \text {,dry }} \times \eta$.

The optical properties ( $\mathrm{n}$ and $\mathrm{k}$ ) of the aerosol as a function of size can then be determined. For computational ease, it is assumed that the initial dry aerosol distribution consists of solid particles of pure ammonium sulfate, and that the aerosols grow by the accumulation of pure water. The optical constants $n$ and $\mathrm{k}$ are then determined using volume fractions;

$$
n_{\text {wet }}=f\left[\left(\mathrm{NH}_{4}\right)_{2} \mathrm{SO}_{4}\right] \times n\left[\left(\mathrm{NH}_{4}\right)_{2} \mathrm{SO}_{4}\right]+f\left[\mathrm{H}_{2} \mathrm{O}\right] \times n\left[\mathrm{H}_{2} \mathrm{O}\right]
$$

and

$$
k_{\text {wet }}=f\left[\left(\mathrm{NH}_{4}\right)_{2} \mathrm{SO}_{4}\right] \times n\left[\left(\mathrm{NH}_{4}\right)_{2} \mathrm{SO}_{4}\right]+f\left[\mathrm{H}_{2} \mathrm{O}\right] \times n\left[\mathrm{H}_{2} \mathrm{O}\right]
$$

where

$$
\begin{aligned}
\mathrm{f}\left[\left(\mathrm{NH}_{4}\right)_{2} \mathrm{SO}_{4}\right] & =\mathrm{V}\left[\left(\mathrm{NH}_{4}\right)_{2} \mathrm{SO}_{4}\right] / \mathrm{V} \text { (wet aerosol), } \\
& \left.=4 / 3 \pi \mathrm{r}_{\text {dry }}{ }^{3} / 4 / 3 \pi \mathrm{r}_{\text {wet }}{ }^{3}=1 / \eta^{3} \text { (since } \mathrm{r}_{\text {wet }}=\eta \mathrm{r}_{\text {dry }}\right) \text {, and } \\
\mathrm{f}\left[\mathrm{H}_{2} \mathrm{O}\right] & =\eta^{3}-1 / \eta^{3} .
\end{aligned}
$$


Values for $\mathrm{n}$ and $\mathrm{k}$ for water and ammonium sulfate, at the four wavelengths that will be considered here (Hale and Querry 1973; Toon et al. 1976) are:

\begin{tabular}{|c|c|c|c|c|}
\hline Wavelength & $\overline{\mathrm{n} \mathrm{nH}_{2} \mathrm{O}}$ & $\mathrm{n}\left(\mathrm{NH}_{4}\right)_{2} \mathrm{SO}_{4}$ & $\mathrm{kH}_{2} \mathrm{O}$ & $\mathrm{k}\left(\mathrm{NH}_{4}\right)_{2} \mathrm{SO}_{4}$ \\
\hline $266 \mathrm{~nm}$ & 1.3565 & 1.55 & $2.75 \mathrm{E}-8$ & $1.0 \mathrm{E}-7$ \\
\hline $355 \mathrm{~nm}$ & 1.3427 & 1.535 & $5.80 \mathrm{E}-9$ & $1.0 \mathrm{E}-7$ \\
\hline $532 \mathrm{~nm}$ & 1.3337 & 1.52 & $1.45 \mathrm{E}-9$ & $1.0 \mathrm{E}-7$ \\
\hline $1064 \mathrm{~nm}$ & 1.3260 & 1.51 & $5.13 \mathrm{E}-9$ & $2.4 \mathrm{E}-6$ \\
\hline
\end{tabular}

\subsection{VOC-based Aerosols}

Values of $\mathrm{n}$ and $\mathrm{k}$ for a model VOC-based hydrophilic organic aerosol were taken from Hess et al. (1998). These aerosols are assumed to grow with humidity just as sulfate aerosols do and so the model described in the previous section was used to predict the changes in VOC-based aerosol optical properties with humidity. The $\mathrm{r}_{0}, \sigma$ and $\mathrm{N}_{\mathrm{o}}$ values of the "dry" CCN distribution were identical with those used for the sulfate aerosol modeling.

\begin{tabular}{|c|c|c|}
\hline Wavelength (nm) & $\mathbf{n}$ & $\mathbf{k}$ \\
\hline 266 & 1.53 & 0.008 \\
\hline 355 & 1.53 & 0.005 \\
\hline 532 & 1.53 & 0.006 \\
\hline 1064 & 1.52 & 0.0115 \\
\hline
\end{tabular}

\subsection{Soot Aerosols}

Values for soot $\mathrm{n}$ and $\mathrm{k}$ were taken from Marley et al. (2001). Values for a log-normal distribution with $\mathrm{r}_{0}=0.234 \mu \mathrm{m}$ and $\sigma=1.5$ were taken from Lapuerta et al. (2003), and $\mathrm{N}_{\mathrm{o}}$ was assumed to be the same as that for sulfate aerosol, i.e., $8.3 \times 10^{6}$ particles $/ \mathrm{m}^{3}$.

\begin{tabular}{|c|c|c|}
\hline Wavelength (nm) & $\mathbf{n}$ & $\mathbf{k}$ \\
\hline 266 & 1.74 & 0.470 \\
\hline 355 & 1.75 & 0.465 \\
\hline 532 & 1.75 & 0.440 \\
\hline 1064 & 1.75 & 0.435 \\
\hline
\end{tabular}




\subsection{Arizona Road Dust Aerosols}

Values for $\mathrm{n}$ and $\mathrm{k}$ were taken from Querry (1987). Values for a log-normal distribution with $\mathrm{r}_{0}=$ $0.25 \mu \mathrm{m}$ and $\sigma=1.5$ were taken from Marple et al. (1978), and $\mathrm{N}_{\mathrm{o}}$ was assumed to be the same as that for sulfate aerosol.

\begin{tabular}{|c|c|c|}
\hline Wavelength (nm) & $\mathbf{n}$ & $\mathbf{k}$ \\
\hline 266 & 1.339 & 0.00692 \\
\hline 355 & 1.339 & 0.00138 \\
\hline 532 & 1.336 & 0.00110 \\
\hline 1064 & 1.332 & 0.00110 \\
\hline
\end{tabular}

\subsection{Bacillus Subtillus var. niger Aerosols}

An electron micrograph picture of B. Subtillus var. niger (also known as B. globigii), taken at PNNL by Dr. Nancy Valentine, shows that the dried cleaned spores look like rods that are about 1 micron in length and 0.5 micron in diameter. For modeling purposes, however, it would most likely be best to consider the spores as prolate ellipsoids, with $\mathrm{a}=\mathrm{b}<\mathrm{c}(\mathrm{a}=\mathrm{b}=0.5 \mu \mathrm{m}, \mathrm{c}=1 \mu \mathrm{m})$ since real biological systems seldom contain sharp edges. The prolate ellipsoid has an equivalent spherical radius of $0.63 \mu \mathrm{m}$.

The electron micrograph shows that the spore size varies. This has been verified by aerodynamic size measurements (Gurton et al. 2001). In these experiments, the laboratory-generated B. Subtillus var. niger spore aerosols were single spores, near mono-disperse. The aerosols were found to follow a log-normal size distribution with an aerodynamic radius, $\mathrm{r}_{\mathrm{o}}=0.89 \mu \mathrm{m}$, and $\sigma=1.5$. The aerodynamic radius is larger than a volume-equivalent spherical radius because rod-shaped particles have added drag. In the calculations, $\mathrm{r}_{\mathrm{o}}=0.63 \mu \mathrm{m}$ and $\sigma=1.5$ were used. It was also assumed that $\mathrm{N}_{\mathrm{o}}=8.3 \times 10^{6} \mathrm{spores} / \mathrm{m}^{3}$, the same as for all other aerosols, to enable comparison of scattering behavior.

Tuminello et al. (1997) have measured the complex refractive index of B. Subtillis spores. The $\mathrm{n}$ and $\mathrm{k}$ values they report for fresh spores in water were used, specifically:

\begin{tabular}{|c|c|c|}
\hline Wavelength (nm) & $\mathbf{n}$ & $\mathbf{k}$ \\
\hline 266 & 1.55 & 0.0138 \\
\hline 355 & 1.53 & 0.0164 \\
\hline 532 & 1.52 & 0.0178 \\
\hline 1064 & 1.52 & 0.0125 \\
\hline
\end{tabular}




\subsection{Erwinia Herbicola Aerosols}

Arakawa et al. (2003) have measured the wavelength-dependent complex refractive index of Erwinia Herbicola, which is a Gram-negative, non-spore forming (i.e., vegetative) bacterium. The table below lists the $\mathrm{n}$ and $\mathrm{k}$ values at the wavelengths at which the Mie scattering calculations were done.

\begin{tabular}{|c|l|l|}
\hline Wavelength (nm) & $\mathbf{n}$ & \multicolumn{1}{|c|}{$\mathbf{k}$} \\
\hline 266 & 1.587 & 0.00337 \\
\hline 355 & 1.546 & 0.000373 \\
\hline 532 & 1.523 & 0.0002 \\
\hline 1064 & 1.51 & 0.0000875 \\
\hline
\end{tabular}

Arakawa reports that Erwinia is rod-shaped with $0.5 \mu \mathrm{m}$ diameter and 1-2 $\mu \mathrm{m}$ length. This system was modeled as a prolate elliposoid with volume equivalent spherical radius of $0.63 \mu \mathrm{m}$ and a log-normal size distribution with $\sigma=1.5 \mu \mathrm{m}$, i.e., the same dimensions as used for modeling B. Subtillus. The calculations were tried with a slightly larger equivalent radius, $0.8 \mu \mathrm{m}$ (that corresponds to a 2- $\mu \mathrm{m}$ length), but problems were encountered with the T-matrix code convergence. 


\subsection{Results}

\subsection{Influence of Humidity on Ambient Aerosol Scattering}

In discussions with JBSDS subject matter experts, it was suggested that the impact that humidity changes have upon elastic backscattering profiles be investigated to see what effect this might have on a LIDAR sensor false-positive rate. Figure 5.1 shows the wavelength-dependent elastic backscatter cross sections that were calculated for sulfate and VOC-based aerosols at 40, 60, and 80\% relative humidity. Independent of humidity, the cross sections for the two systems monotonically decrease with increasing excitation wavelength. The imaginary part of the sulfate and VOC compound refractive index increase with increasing wavelength, meaning that absorption will compete with elastic backscattering as the excitation wavelength moves to the red end of the spectrum. In addition, the real part of the refractive index in both systems decreases with increasing wavelength, which will also cause a decrease in elastic backscattered intensity.

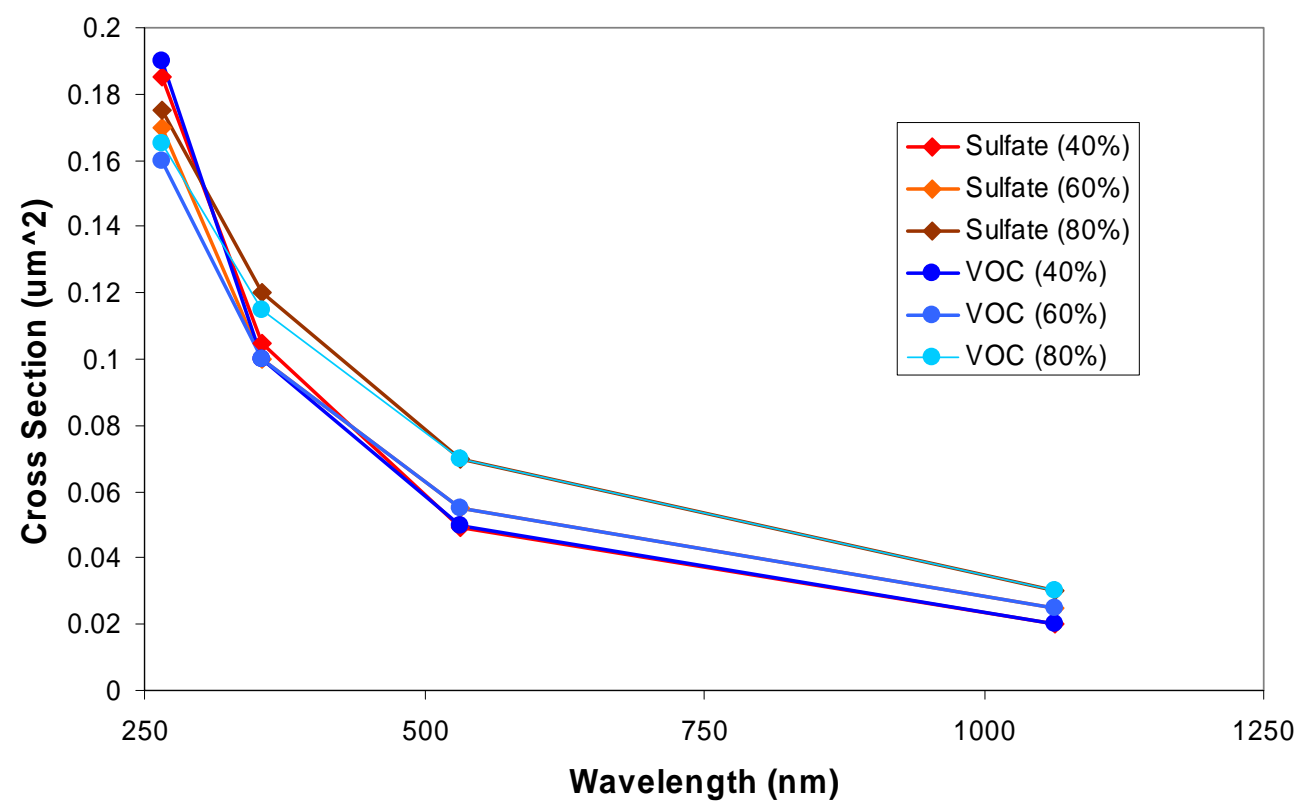

Figure 5.1. Elastic Backscatter Cross Sections Calculated for Sulfate and VOC Aerosol Systems as a Function of Relative Humidity

Surprisingly enough, humidity has only a marginal effect on the elastic scattering cross sections in both systems. At $1064 \mathrm{~nm}$, the elastic backscatter cross sections increase by $50 \%$ when the humidity is doubled; i.e., goes from $40 \%$ relative humidity $(\mathrm{RH})$ to $80 \% \mathrm{RH}$. This same type of behavior is seen for scattering at 532 and $355 \mathrm{~nm}$, but the effect is not as pronounced; i.e., the $\sigma$ 's increase only 15\% when the humidity is doubled. The observation of increased scatter efficiency with increased humidity obviously stems from an increase in the particle diameters as they grow through water uptake. At $266 \mathrm{~nm}$, however, the backscattering cross sections decrease with increasing $\mathrm{RH}$, by around $5 \%$. While the increase in 
humidity will cause an increase in particle size, the growth is generated by water uptake. The water absorption cross section increases with transition into the UV, so this most likely is what is causing the decrease in backscatter intensity.

\subsection{Elastic Backscattering Profiles of Biological and Non-biological Aerosols}

The backscatter cross sections that were calculated for B. Subtillus, Erwinia Herbicola, sulfate (60\% relative humidity), VOC-based (60\% relative humidity), soot and Arizona road dust aerosols at 266, 355, 532, and 1064 are listed in Table 5.1 and plotted in Figure 5.2.

Table 5.1. Calculated Elastic Backscatter Cross Sections in $\mu \mathrm{m}^{2}$

\begin{tabular}{|c|c|c|c|c|c|c||}
\hline $\begin{array}{c}\text { Wavelength } \\
(\mathbf{n m})\end{array}$ & B. Subtillus & $\begin{array}{c}\text { Erwinia } \\
\text { Herbicola }\end{array}$ & $\begin{array}{c}\text { Sulfate } \\
(\mathbf{6 0 \%} \mathbf{R H})\end{array}$ & $\begin{array}{c}\text { VOC } \\
\mathbf{( 6 0 \%} \mathbf{R H})\end{array}$ & Soot & $\begin{array}{c}\text { Arizona } \\
\text { Road Dust }\end{array}$ \\
\hline 266 & 0.33 & 0.92 & 0.17 & 0.16 & 0.026 & 0.115 \\
\hline 355 & 0.43 & 1.30 & 0.10 & 0.10 & 0.027 & 0.11 \\
\hline 532 & 0.62 & 1.11 & 0.055 & 0.055 & 0.028 & 0.078 \\
\hline 1064 & 0.67 & 0.78 & 0.025 & 0.025 & 0.035 & 0.038 \\
\hline
\end{tabular}

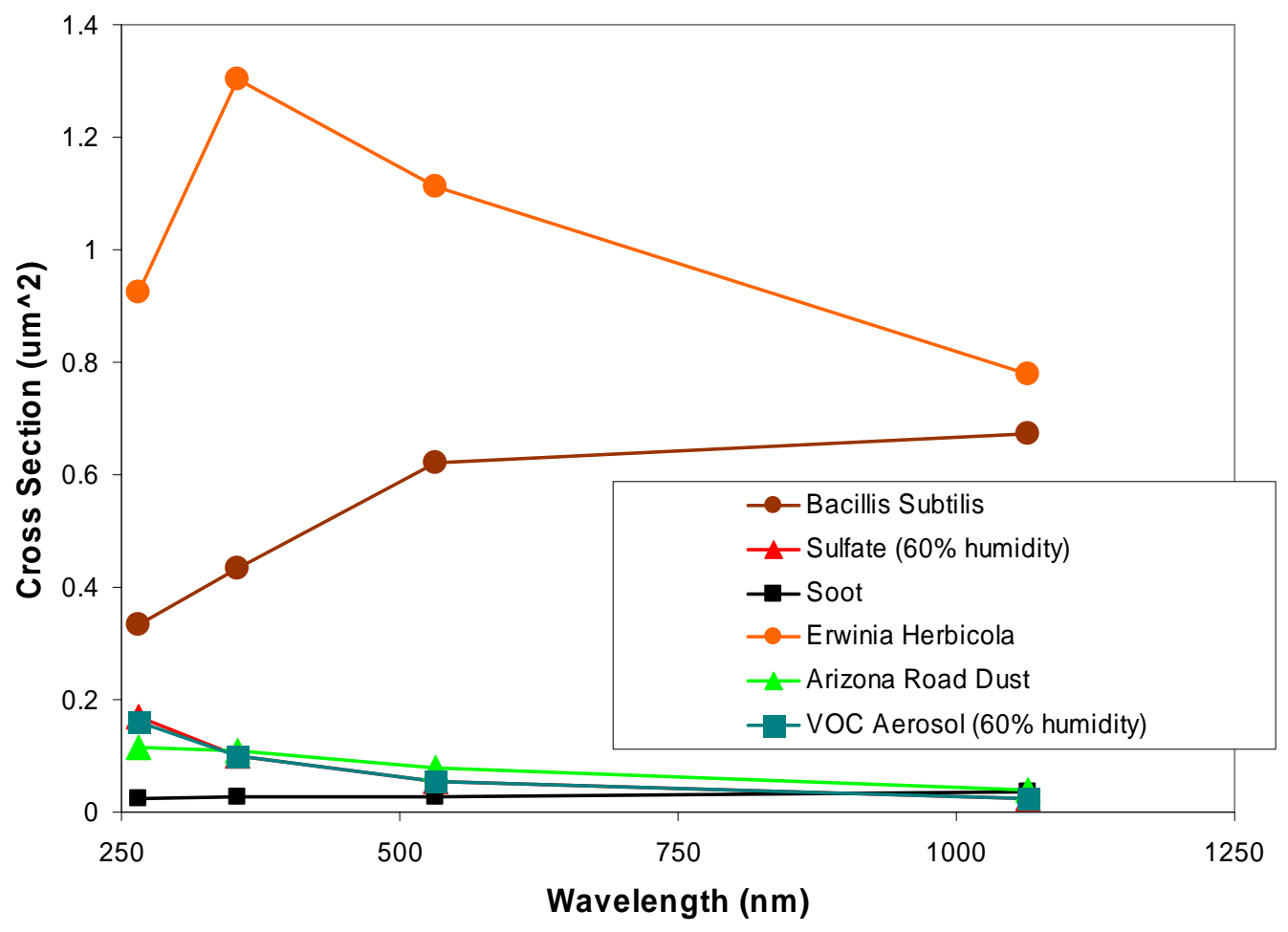

Figure 5.2. Elastic Backscatter Cross Sections for the Different Aerosol Systems 
From Figure 5.2 it can be seen that elastic backscattering off biological particles (both sporulated and vegetative) is much more efficient than scattering off ambient sulfate, VOC-based, soot and road dust particles. The biological system cross sections are a minimum $100 \%$ larger at $266 \mathrm{~nm}$, and $2000 \%$ larger at $1064 \mathrm{~nm}$ than those for the ambient species. A priori, the biological aerosol system backscatter coefficients are expected to be larger, simply because their particle diameters are at least a factor of two bigger than the ambient aerosol counterparts.

Obviously, introducing a cloud of biological aerosols will increase backscattering observed in the field. But if the number density of the "new" cloud is fairly low, it may be difficult to tell if the increased scatter is due to introduction of new material or simply due to increase scatter observed from ambient sulfate or VOC-based aerosols that have grown as a result of an increase in local humidity. It is noted from Figure 5.2, however, that the wavelength response of elastic backscatter is different for the different types of aerosol systems. This is emphasized in Figure 5.3, which plots the relative elastic backscatter cross sections as a function of wavelength.

Figure 5.3 shows that the elastic backscatter from ambient sulfate, VOC-based, and road dust aerosols decreases with increasing wavelength. In contrast, the scattering from the B. Subtillus spores and from soot increases with wavelength, and scattering from Erwinia Herbicola (a vegetative bacteria) first increases and then decreases as the wavelength changes from 266 to $1064 \mathrm{~nm}$. It is clear that by measuring scattering intensities from four wavelengths some form of aerosol categorization will be possible. Certainly sulfates, VOC-based, and road dust aerosols can be immediately categorized. It will be more difficult to categorize the vegetative and sporulated bacteria from each other, or from soot particles.

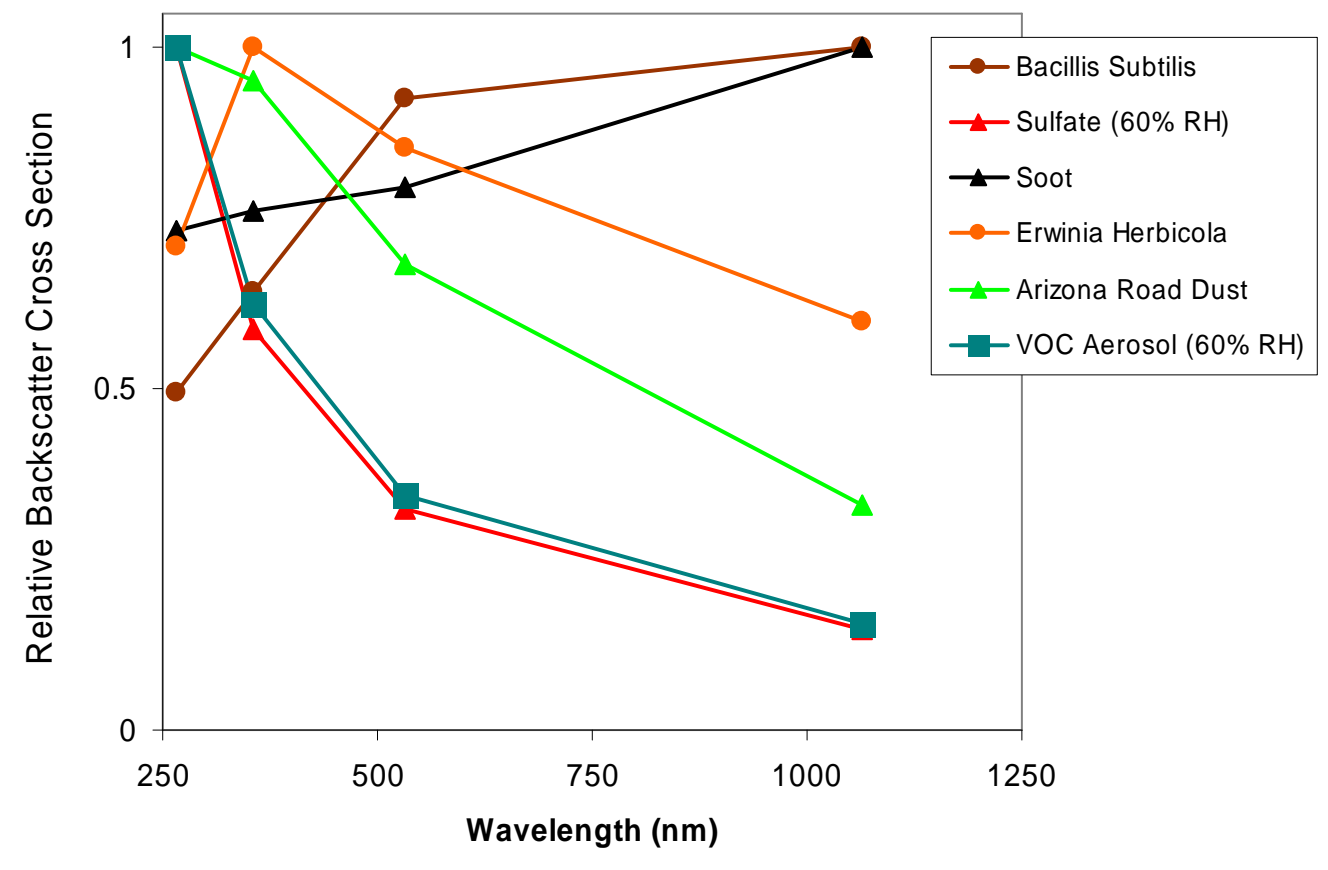

Figure 5.3. Wavelength Dependence of Relative Elastic Backscatter Cross Sections 
5.4 


\subsection{Conclusions}

The calculations show that a four-wavelength elastic-backscatter LIDAR experiment could allow an operator to immediately recognize when sulfate, VOC-based, or road dust (silicate) aerosols are approaching, independent of humidity changes. Separation of biological system aerosols from soot will be more challenging; however, the false-positive detection rate may be lowered by combining multiplewavelength elastic backscatter data with spectrally resolved UV fluorescence profiles - soot fluorescence is very broadband and extends far into the red, whereas emission from biological warfare agents goes out only to about $550 \mathrm{~nm}$.

It must be emphasized that the observations made here are based only on theoretical calculations. While the $\mathrm{n}$ and $\mathrm{k}$ values for sulfate, water, silicate, and soot are well established, those for VOCs and biological systems are still considered tenuous. Obviously, the theoretical results generated in this study need to be verified by doing four-wavelength elastic backscattering field experiments. 
6.2 


\subsection{References}

Agranovski V, Z Ristovski, M Hargreaves, PJ Blackall and L Morawska. 2003. "Real-time measurement of bacterial aerosols with the UVASP: performance evaluation.” J Aerosol Sci 34(3):301-317.

Alimova A, A Katz, HE Savage, M Shah, G Minko, DV Will, RB Rosen, SA McCormick and RR Alfano. 2003. "Native fluorescence and excitation spectroscopic changes in B. subtilis and Staphylococcus aureus bacteria subjected to conditions of starvation.” Appl Opt 42(19):4080-4087.

Arakawa ET, PS Tuminello, BN Khare and ME Milhan. 2003. "Optical Properties of Erwinia herbicola Bacteria and 0.190 - 2.50 mm.” Biopolymers 72:391-398.

Atkins J, ME Thomas and RI Joseph. 2007. "Spectrally resolved fluorescence cross sections of BG and BT with a 266 nm pump wavelength.” Proc. SPIE 6554:6554OT-1 - 6554OT-8.

Bohren CF and DR Huffman. 1983. Absorption and Scattering of Light by Small Particles. John Wiley and Sons, New York.

Bronk BV and L Reinisch. 1991. "Variability of steady-state bacterial fluorescence with respect to growth conditions.” Appl Spectrosc 47:436-440.

Brosseau LM, D Vesley, N Rice, K Goodell, M Nellis and P Hairston. 2000. "Differences in Detected Fluorescence Among Several Bacterial Species Measured with a Direct-Reading Particle Sizer and Fluorescence Detector.” Aerosol Sci and Tech 32:545-558.

Bucholtz A. 1995. "Rayleigh-scattering calculations for the terrestrial atmosphere.” Appl Opt 34(15):2765-2773.

Cheng YS, EB Barr, BJ Fan, PJ Hargis, DJ Rader, TJ O’Hern, JR Torczynski, GC Tisone, BL Preppernau, SA Young and RJ Radloff. 1999. "Detection of Bioaerosols Using Multiple Wavelength UV Fluorescence Spectroscopy.” Aerosol Sci and Tech 30:186-201.

Dalterio RA, WH Nelson, D Britt, J Sperry, D Pasaras, JF Tanqua and SL Suib. 1987. "Steady-state and decay characteristics of protein tryptophan fluorescence from bacteria.” Appl Spectrosc 41:234-241.

Farris GW, RA Copeland, K Mortelmans and BV Bronk. 1997. "Spectrally resolved absolute fluorescent cross sections for bacillus spores.” Appl Opt 36(4):958-967.

Fell NF, RG Pinnick, SC Hill, G Videen, S Niles, RK Chang, S Holler, Y Pan, JR Bottiger and BV Bronk. 1998. "Concentration, Size and Excitation Power Effects on Fluorescence from Microdroplets Containing Tryptophan and Bacteria.” Proc. SPIE 3533:52.

Gurton KP, D Ligon and R Kvavilashivili. 2001. "Measured infrared spectral extinction for aerosolized Bacillus subtilis var. niger endospores from 3 to 13 mm.” Appl Opt 40(25):4443-4448. 
Hale GM and MR Querry. 1973. “Optical Constants of Water in the $200 \mathrm{~nm}$ to $200 \mathrm{Om}$ Wavelength Region.” Appl Opt 12(3):557.

Halverson J, RK Chang, Y-L Pan, J Harting, SC Hill and RG Pinnick. 2003. "Fluorescent Particle Spectrometer for Measuring Single Shot Fluorescence Spectra of Single Particles in Ambient Air.” Aerosol Sci and Tech 37(8):628-639.

Hargis PJ, GC Tisone, JS Wagner, TD Raymond and TL Downey. 1994. "Multispectral ultraviolet fluorescence Lidar for environmental monitoring.” Proc. SPIE 2366:394-400.

Hess M, P Koepke and I Schult. 1998. “Optical Properties of Aerosols and Clouds.” B Am Meteorol Soc 79(5):831-850.

Hill SC, RG Pinnick, S Niles, NF Fell, Y-L Pan, JR Bottiger, BV Bronk, S Holler and RK Chang. 2001. "Fluorescence from airborne microparticles: dependence on size, concentration of fluorophores, and illumination intensity." Appl Opt 40(18):3005-3011.

Hill SC, RG Pinnick, S Niles, Y-L Pan, S Holler, RK Chang, JR Bottiger, BT Chen, C-S Orr and G Feather. 1999. "Real-Time Measurement of Fluorescence Spectra from Single Airborne Biological Particles.” Field Anal Chem Tech 3(4-5):221-229.

Johnson DL, TA Pearce and NA Esmen. 1999. "The effect of phosphate buffer on aerosol size distribution of nebulized Bacillus subtilis and Pseudomonas fluorescens Bacteria." Aerosol Sci and Tech 30:202-210.

Kong JA. 1986. Electromagnetic Wave Theory. John Wiley and Sons, New York.

Kunnil J, S Sarasanandarajah, E chacko and L Reinisch. 2005. "Fluorescence quantum efficiency of dry Bacillus globigii spores.” Opt Express 13(22):8969-8979.

Lapuerta M, O Armas and A Gomex. 2003. “Diesel Particle Size Distribution Estimated from Digital Image Analysis.” Aerosol Sci and Tech 37:369-381.

Li J, JGD Wong, JS Dobbie and P Chylek. 2001. "Parameterization of the optical properties of sulfate aerosols.” J. Atmos. Sci 58:193-209.

Marley NA, JS Gaffney, JC Baird, CA Blazer, PJ Drayton and JE Frederick. 2001. “An Empirical Method for the Determination of the Complex Refractive Index of Size-Fractionated Atmospheric Aerosols for Radiative Transfer Calculations.” Aerosol Sci and Tech 34:535-549.

Marple VA, BYH Liu and K Rubow. 1978. “A dust generator for laboratory use.” Am Ind Hyg Assoc J 39(1):26-32.

Measures RM. 1984. Laser Remote Sensing. John Wiley and Sons, New York. 
Mishchenko MI, LD Travis and AA Lacis. 2002. Scattering, Absorption, and Emission of Light by Small Particles. Cambridge University Press, Cambridge (UK).

National Research Council. 2005. Sensor Systems for Biological Agent Attacks: Protecting Buildings and Military Bases. Committee on Materials and Manufacturing Processes for Advanced Sensors, Board on Manufacturing and Engineering Design, Division on Engineering and Physical Sciences, National Research Council of the National Academies. The National Academic Press, Washington, DC.

Nudelman R, BV Bronk and S Efrima. 2000. "Fluorescence Emission Derived from Dipicolinic Acid, its Sodium, and its Calcium Salts.” Appl Spectrosc 54(3):445-449.

Pan Y-L, S Holler, RK Chang, SC Hill, RG Pinnick, S Niles and JR Bottiger. 1999. "Single shot fluorescence spectra of individual micro-sized bio-aerosols illuminated by a $351 \mathrm{~nm}$ or $266 \mathrm{~nm}$ laser." Optics Letters 24(2):116-118.

Press WH, SA Teukolsky, WT Vetterling and BP Flannery. 1992. Numerical Recipes in C, Second Edition. Cambridge University Press, Cambridge (UK).

Querry MR. 1987. Optical constants of minerals and other materials from the millimeter to the ultraviolet. CRDEC-CR88009. Chemical Research, Development, and Engineering Center, Aberdeen Proving Grounds, MD.

Sarasanandarajah S, J Kunnil, BV Bronk and L Reinisch. 2005a. "Two-dimensional multiwavelength fluorescence spectra of dipicolinic acid and calcium dipicolinate.” Appl Opt 44(7):1182-1187.

Sarasanandarajah S, J Kunnil, E Chacko, BV Bronk and L Reinisch. 2005b. "Reversible changes in fluorescence of bacterial endospores found in aerosols due to hydration/drying." J Aerosol Sci 36:689699.

Seaver M, JD Eversole, JJ Hardgrove, WK Cary and DC Roselle. 1999. "Size and Fluorescence Measurements of Field Detection of Biological Aerosols.” Aerosol Sci and Tech 30:174-185.

Seaver M, DC Roselle, JF Pinto and JD Eversole. 1998. "Absolute emission spectra from B. subtilis and E. coli vegative cells in solution.” Appl Opt 37(22):5344-5347.

Shelly DC, JM Quarles and IM Warner. 1980a. "Identification of fluorescent Pseudomonas species.” Clin Chem 26:1127-1132.

Shelly DC, JM Quarles and IM Warner. 1980b. "Multiparameter approach to the "fingerprint” of fluorescent Pseudomonas.” Clin. Chem. 26:1419-1424.

Sindoni OI, R Saija, MA Iati, F Borghese, P Denti, GE Fernandes, Y-L Pan and RK Chang. 2006. "Optical scattering by biological aerosols: experimental and computational results on spore simulants." Opt Express 14(15):6942-6950. 
Sivaprakasam V, AL Huston, C Scotto and JD Eversole. 2004. "Multiple UV wavelength excitation and fluorescence of bioaerosols.” Opt Express 12:4457-4466.

Sorrell MJ, J Tribble, L Reinisch, JA Werkhave and RH Ossoff. 1994. "Bacteria identification of otitis media with fluorescence spectroscopy.” Lasers Surf. Med. 14:155-163.

Stephens JR. 1998. Measurements of the UV Fluorescence Cross Sections and Spectra of Bacillus anthrasis Simulants. Final Report for US Army CBDCOM. ERDEC.

Taylor K and JE Penner. 1994. "Response of the climate system to atmospheric aerosols and greenhouse gases.” Nature 369:734-737.

Thomas ME and MB Airola. 2007. "Extinction and backscatter cross sections of biological materials.” Proc. SPIE 6554:65540Q1-65540Q7.

Toon OB, JB Pollack and BN Khare. 1976. "The optical constants of several atmospheric aerosol species: Ammonium sulfate, ammonium oxide and sodium chloride.” J Geophys Res 81(33):5733.

Tuminello PS, ET Arakawa, BN Khare, JM Wrobel, MR Querry and ME Milhan. 1997. “Optical properties of Bacillus subtilis spores from 0.2 to 2.5 mm.” Appl Opt 36(13):2818-2824.

Warren JW, ME Thomas, EW Rogala, A Maret, CA Schumacher and A Diaz. 2004. "Systems Engineering Tradeoffs for a Bio-Aerosol Lidar Referee System.” Proceedings of SPIE 5416:202-215.

Wichert R, W Klemm, K Legenhausen and C Pawellek. 2002. "Determination of Fluorescence CrossSectons of Biological Aerosols.” Part Part Syst Char 19:216-222. 


\section{Appendix A}

Modeling Code Description - MatLab 



\section{Appendix A}

\section{Modeling Code Description - MatLab}

The main graphical user interface (GUI) that controls all of the Bio-Spectral Algorithm Stimulator (BSAS) operations is bs a $s_{-} g u i$. m. This code is executed simply by typing ' bs a s $g u i$ ' from within the MatLab Command Window. A screen capture showing the operation of this GUI is shown in Figure A.1. Other GUIs that can be spawned from within bs a s g u i from the Run menu include aerosol_bsas_gui.m, fascod_sas_gui.m, spectravi ewer. m, and wave. m. These codes are described briefly below, and screen captures of each are shown in Figures A.2-A.5. The bs a s_ gui . $\mathrm{m}$ program features include the File menu, which provides the ability to load and save the BSAS calculation configurations (all computed results are saved to a MatLab *.mat file). The Run menu controls the spawning of the additional GUI tools. The Plot menu provides the ability to plot essentially all of the important spectra or waveforms used by, or computed by, the BSAS. The View menu provides similar access to text and numerical results that are not well suited to plotting for visualization.

The control of the BSAS follows a left-to-right top-to-bottom flow. The Range button spawns the dialog box shown in Figure A.6, which controls the range parameter definitions. The Bkg Aero button spawns the dialog box shown in Figure A.7, which controls the atmospheric visibility parameters. The LIDAR button creates the dialog box shown in Figure A.8 and controls the LIDAR size and energy parameters. The Aero button (Figure A.9) allows selection of a pre-computed aerosol cross-section set. The Plume button spawns the dialog box shown in Figure A.10 and controls the size and density of the bio-aerosol plume. The Atmos button opens the dialog box shown in Figure A.11, and allows loading of a pre-computed atmospheric transmission spectrum. The Trans button opens the dialog box shown in Figure A.12 and allows modification of the atmospheric transmission (overrides the value obtained from the loaded spectrum). The LIDAR System, Telescope, Detector, and Scenario buttons open the dialog boxes shown in Figures A.13-A.16, and define the technical parameters needed for a detailed signal-tonoise ratio calculation for the LIDAR system. Note that this calculation, while performed, is not integrated into the current version of the BSAS. Results obtained from the BSAS calculation (subsequent to pushing the Calculate button) are shown in Figures A.17-A.22. 


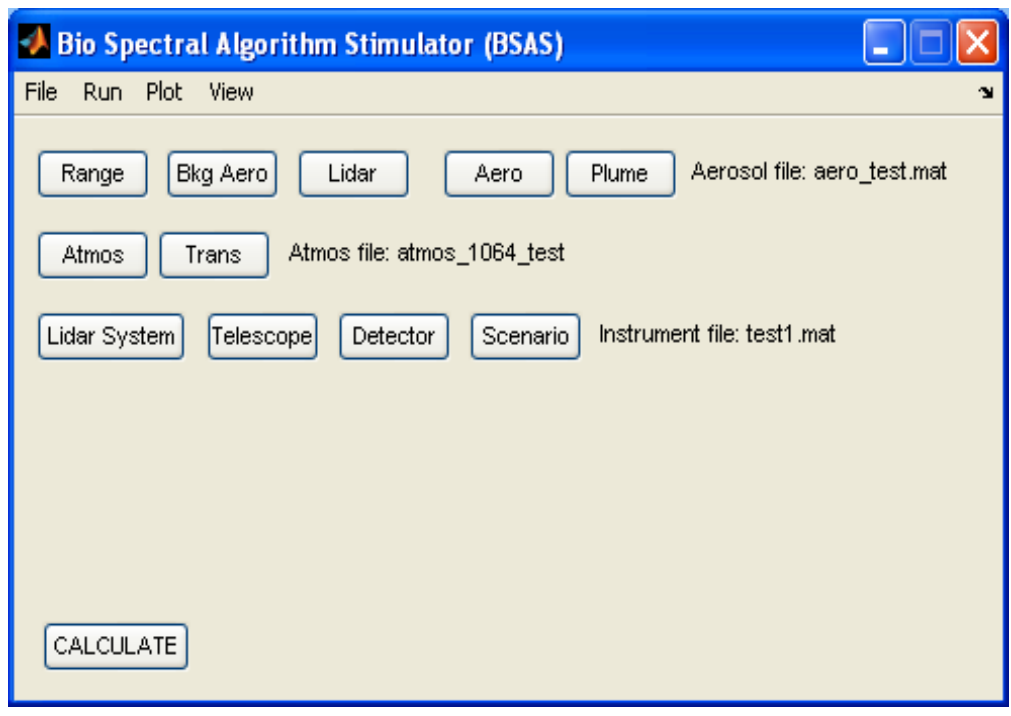

Figure A.1. Screen Capture of b s a s _ g u i . $m$ - the Main BSAS GUI Panel

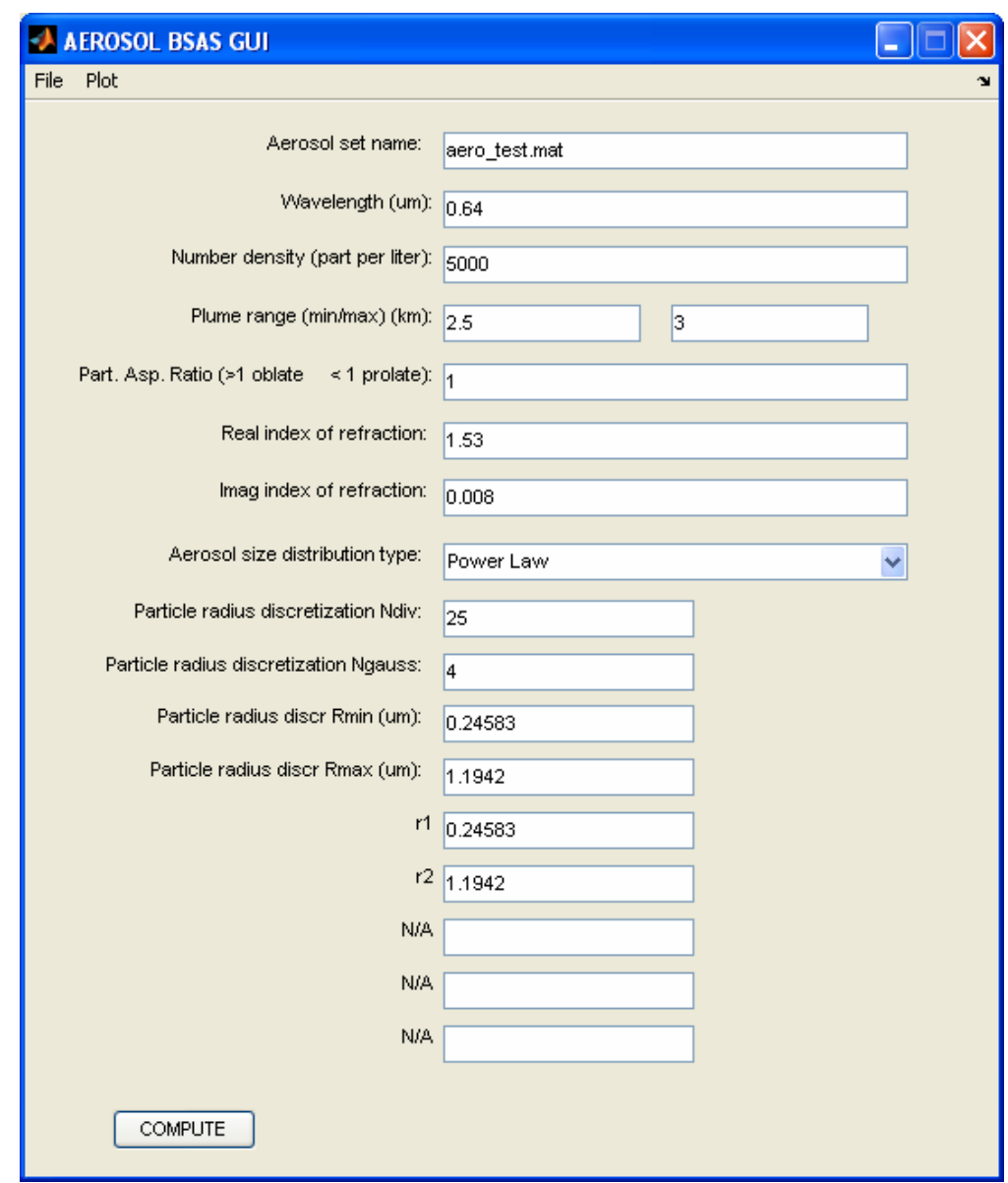

Figure A.2. Screen Capture of a e r o $s_{0} l_{\text {_ }}$ bs a s _ gui . $m$ - the Aerosol BSAS GUI Panel 


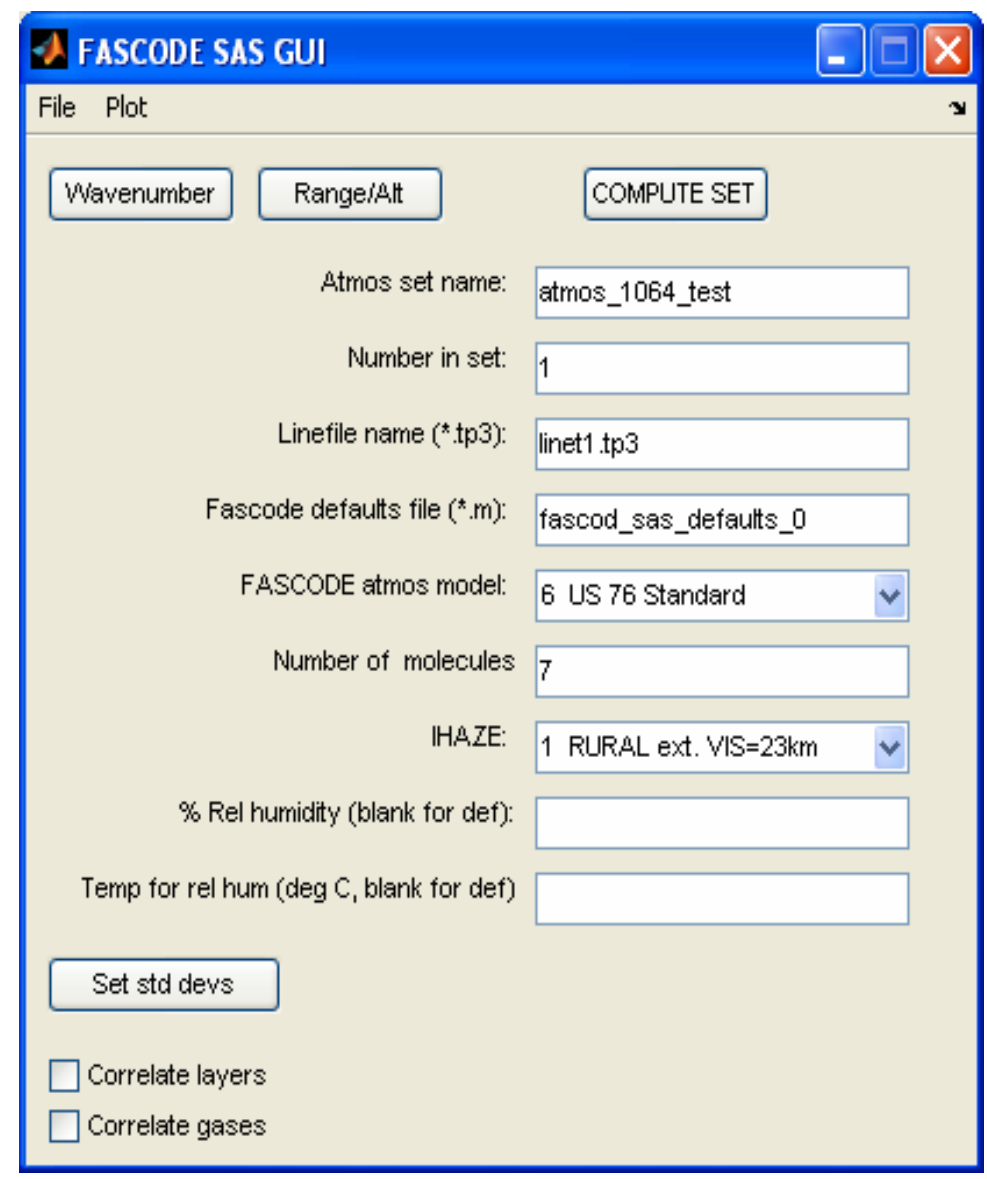

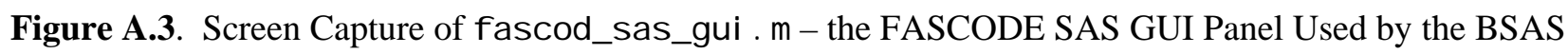

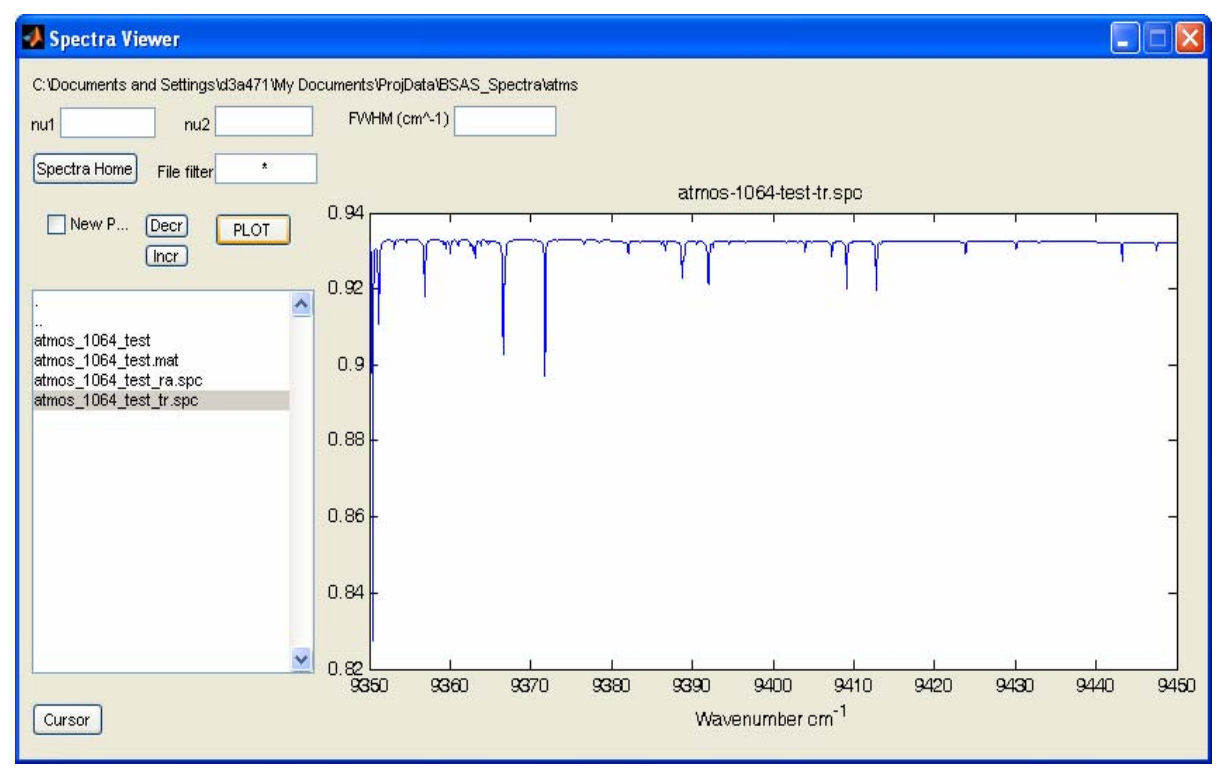

Figure A.4. Screen Capture of s pect $r$ a v i e we r . m - a Spectral Viewing GUI Utility 


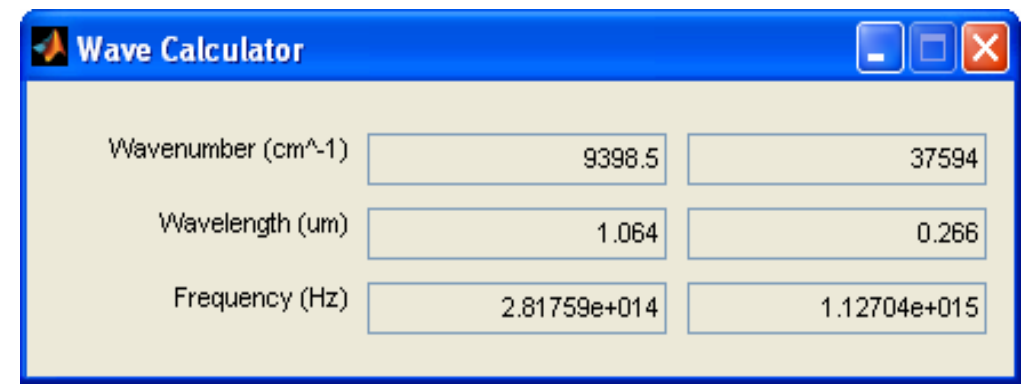

Figure A.5. Screen Capture of wa v e . $m$ - a Calculator for Converting between Wavelength, Wavenumber, and Frequency

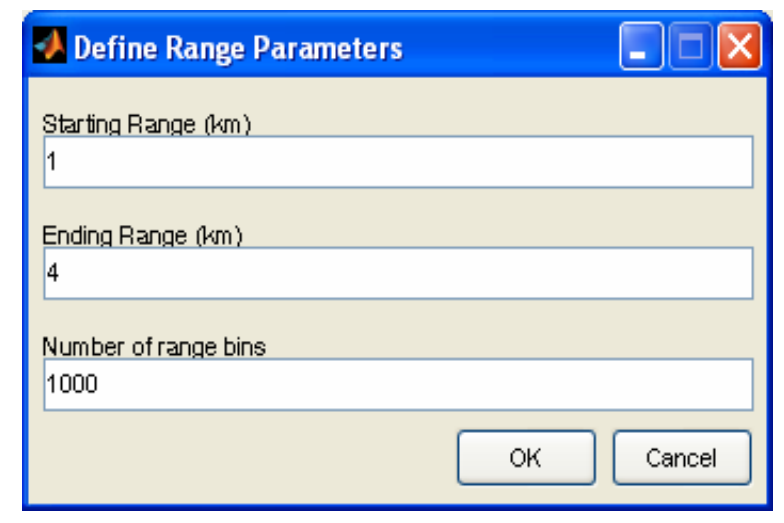

Figure A.6. Range Button Dialog Box

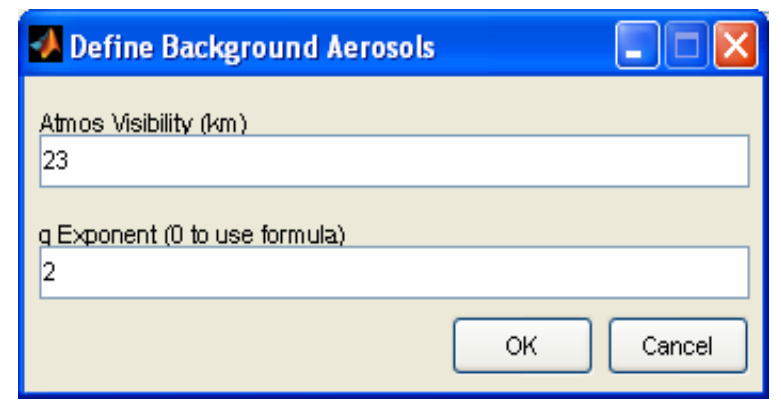

Figure A.7. Bkg Aero Button Dialog Box 


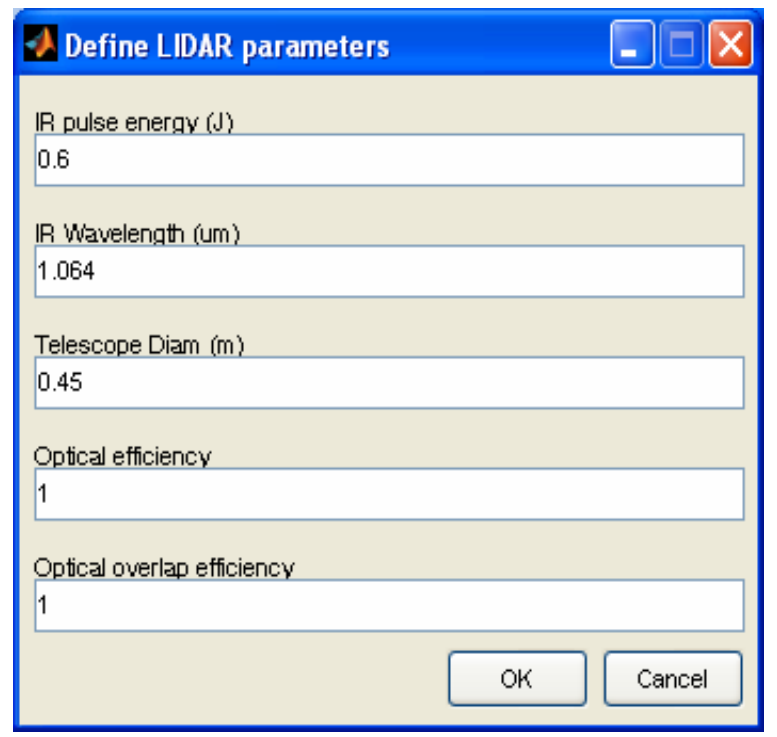

Figure A.8. LIDAR Button Dialog Box

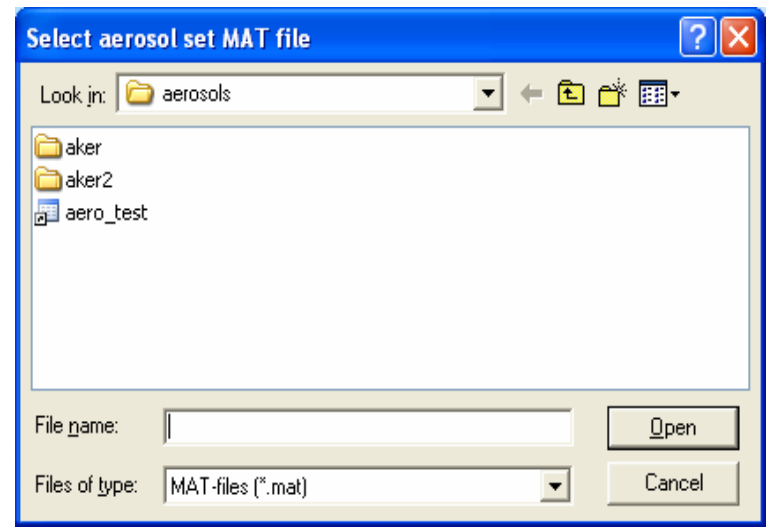

Figure A.9. Aero Button Dialog Box

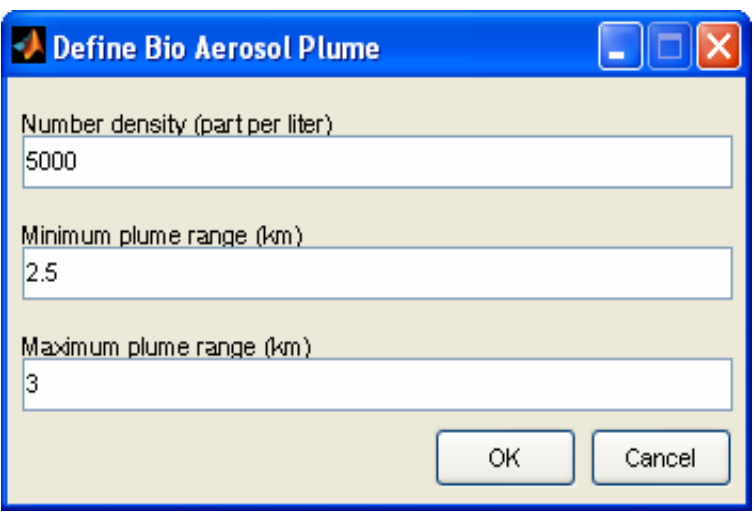

Figure A.10. Plume Button Dialog Box 


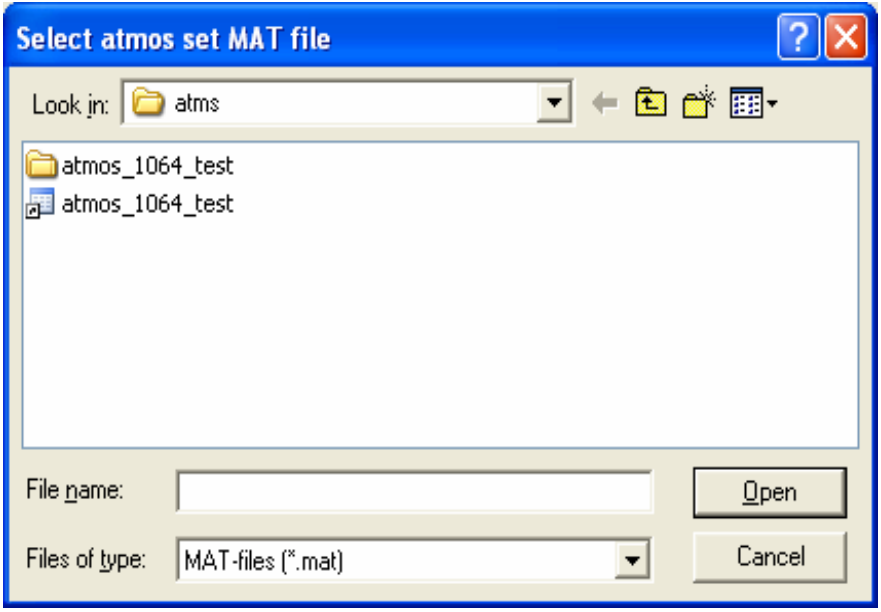

Figure A.11. Atmos Button Dialog Box

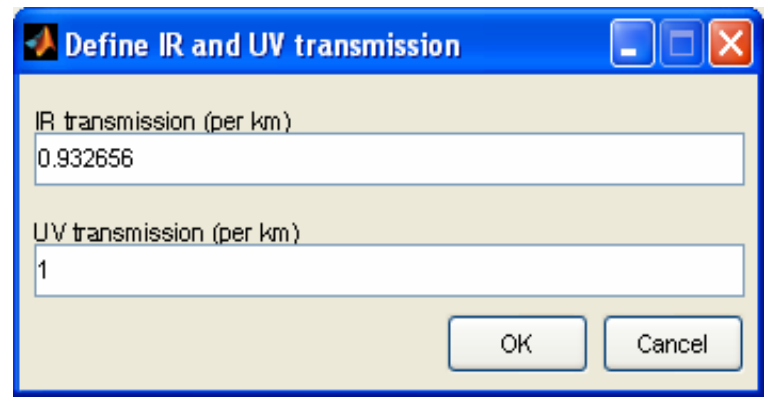

Figure A.12. Trans Button Dialog Box

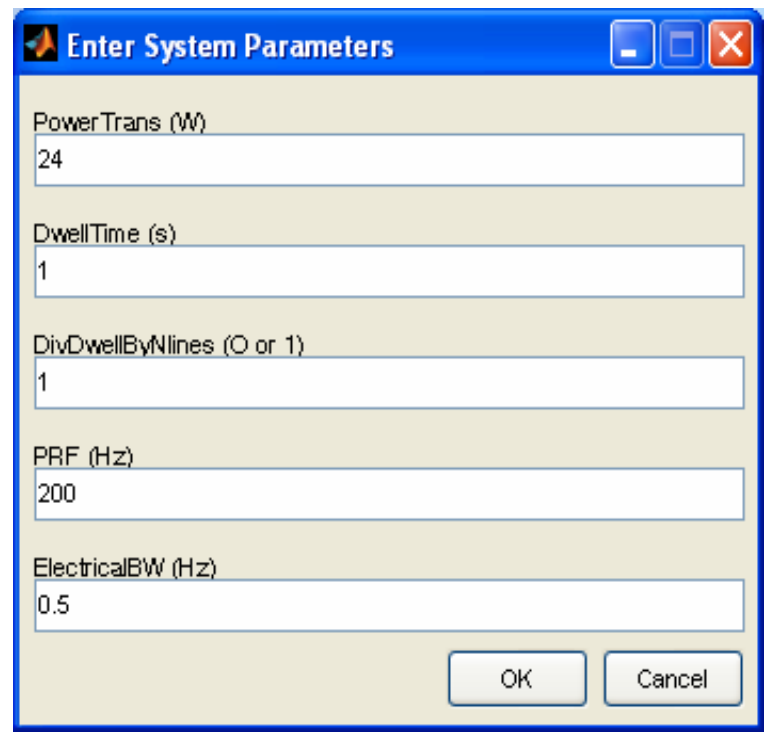

Figure A.13. LIDAR System Button Dialog Box 


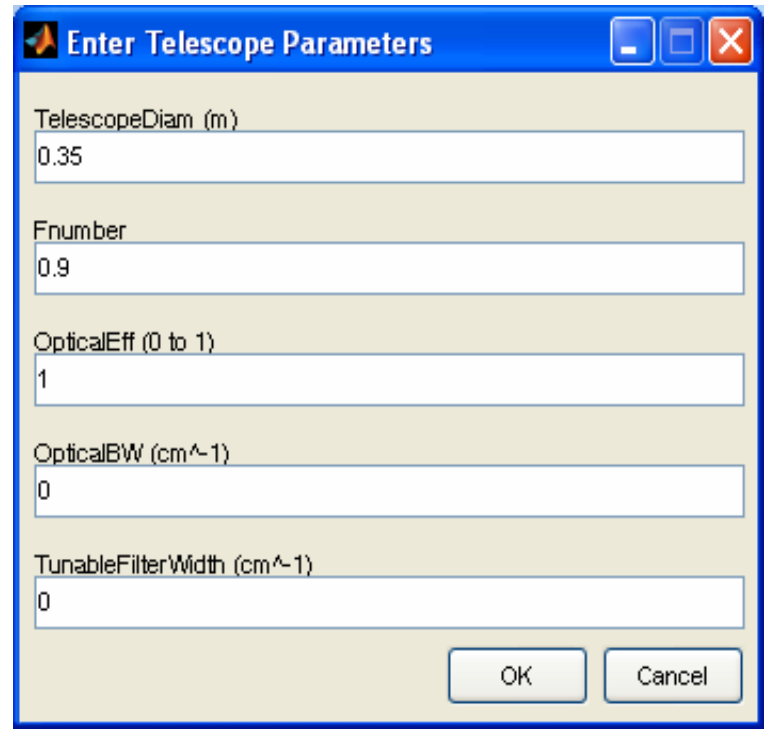

Figure A.14. Telescope Button Dialog Box

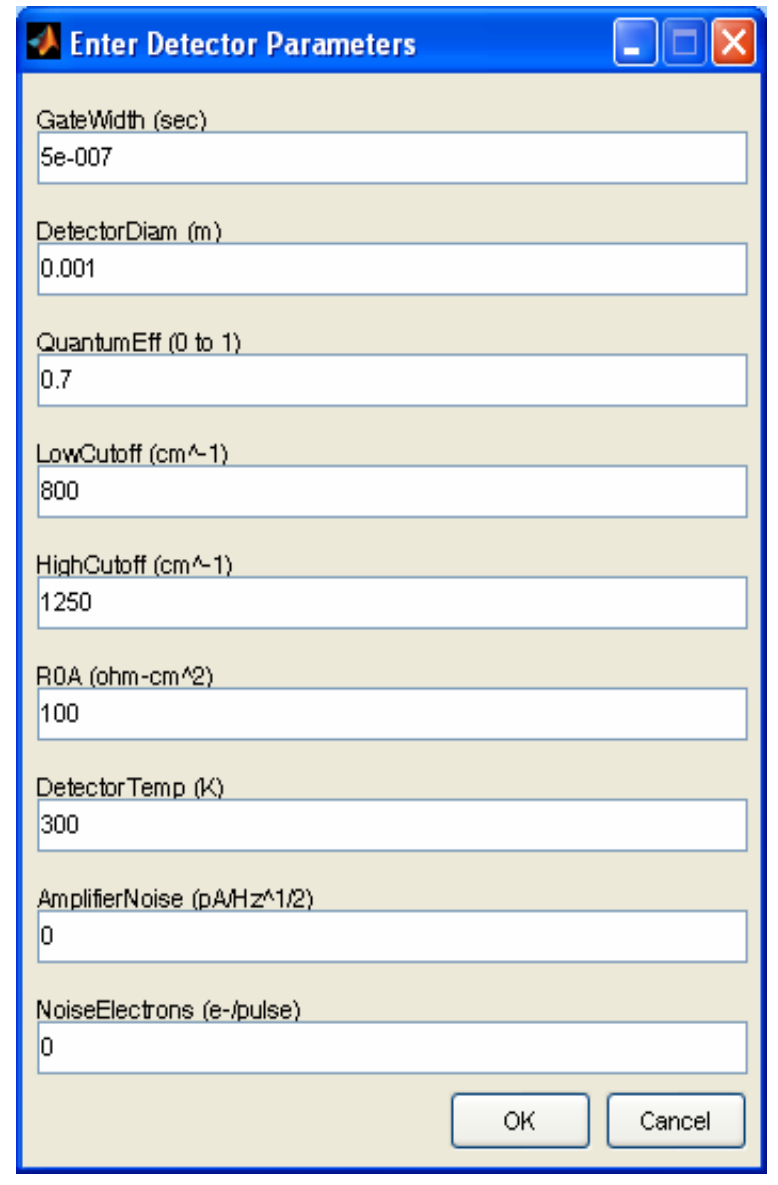

Figure A.15. Detector Button Dialog Box 


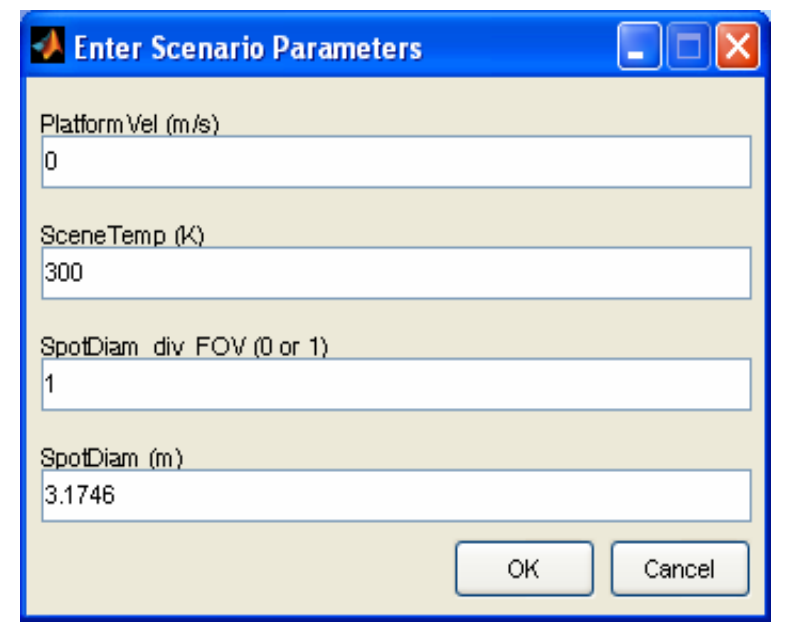

Figure A.16. Scenario Button Dialog Box

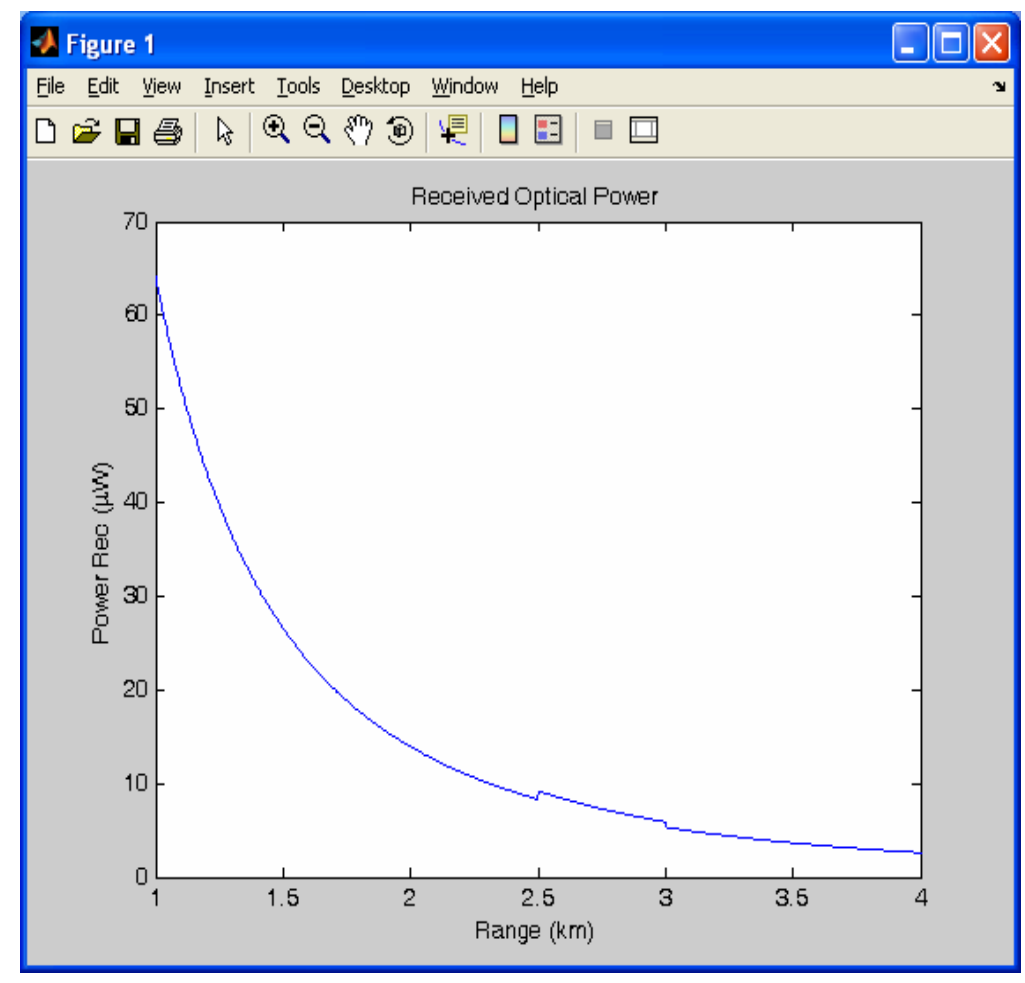

Figure A.17. Received Optical Power for the Parameters in the Saved BSAS file: $t$ es $t$ _ $b s$ a . mat ) 


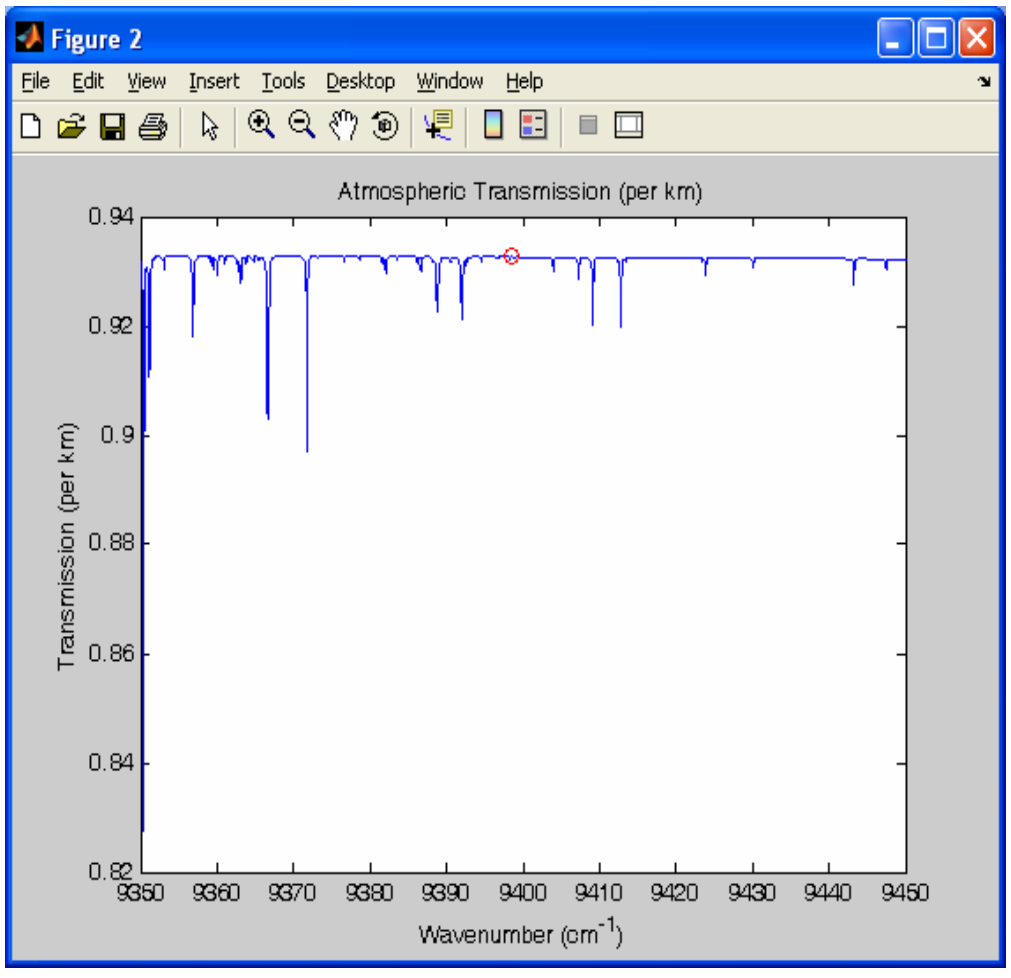

Figure A.18. Atmospheric Transmission for the Parameters in the Saved BSAS file: $t$ e s t _ bs a s . ma t

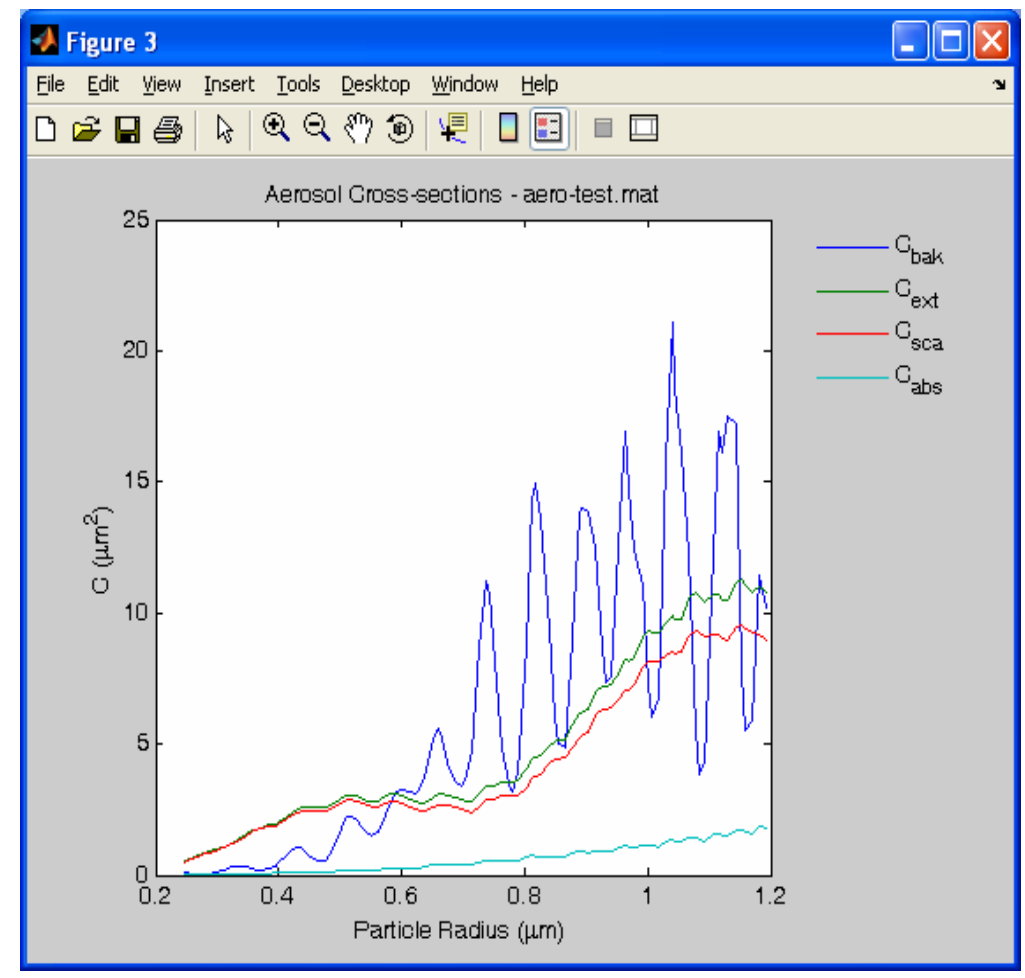

Figure A.19. Aerosol Cross Sections for the Parameters in the Saved BSAS file: $t$ e s t _ bs a s. mat 


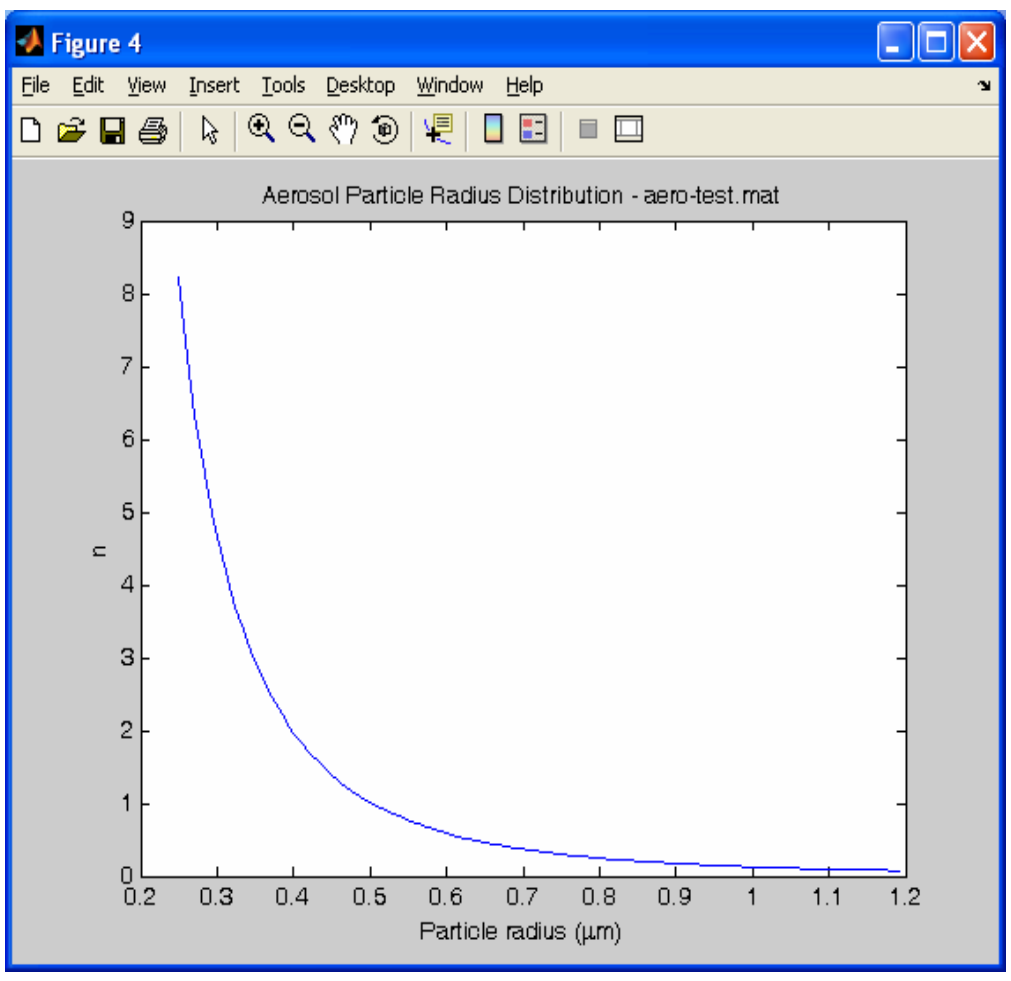

Figure A.20. Aerosol Particle Size Distribution for the Parameters in the Saved BSAS file: $t$ es $t$ _ b s a s. mat

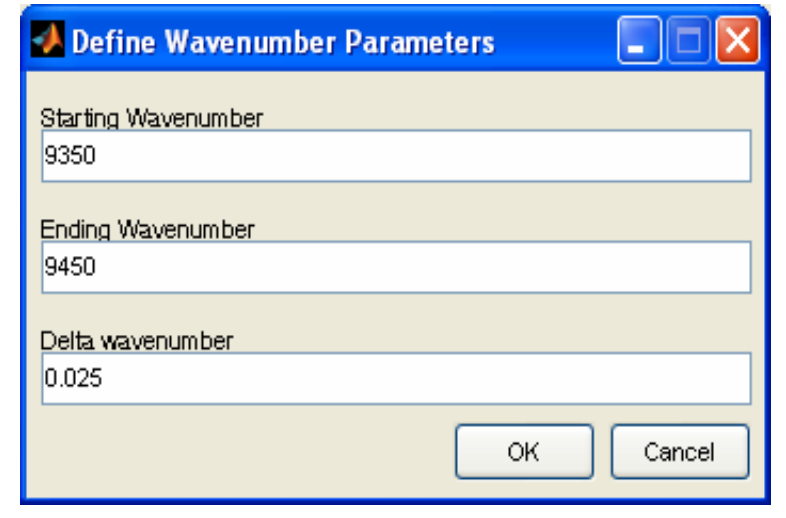

Figure A.21. Wavenumber Parameters Dialog Box for a e r o s ol _ bs a s_ gui . m 


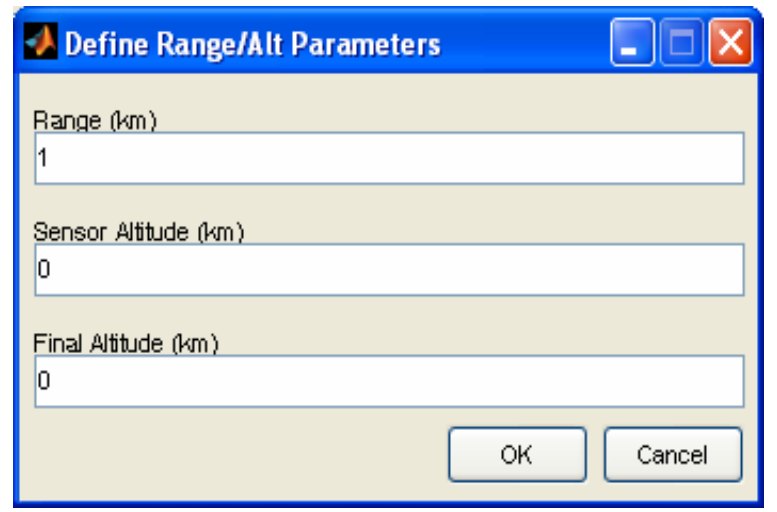

Figure A.22. Range/Alt Parameters Dialog Box for a er os ol _ bs a s_ gui . m

\section{A.1 List of BSAS Matlab Functions}

\section{A.1.1 BSAS Graphical User Interfaces (GUI’s)}

bsas gui.m - is the main graphical user interface (GUI) for the Bio-spectral Algorithm Stimulator (BSAS). It was developed using Matlab's GUIDE tool so some of the code has been auto-generated.

I/0: bsas_gui or bsas_gui( i n )

in i $\bar{s}$ the BSAS däta structure

aerosol bsas_gui.m - is a GUI to control aerosol spectra calculations for the BSAS. The code was created using Matlab's GUIDE tool, so some of the code has been automatically generated.

1/0: aerosol_bsas_gui or aersol_bsas_guil (aero)

aero is ${ }^{-}$the $\bar{B} S A S$ aerosol data structure ( see

default_bsasaerosol_struct. m)

fascod_sas_gui.m - is the GUI for the SAS fascode driver program

( fascos $s a \bar{s} . m$ ). The code was created using Matlab's GUIDE tool, so some of the codé has been automatically generated.

1/0: fascod_sas_gui or fascod_sas_gui (at m) at $\mathrm{m}$ i s ${ }^{-}$the ${ }^{-}$BSAS at mosphe $\bar{r}$ ic $\bar{d}$ at a structure ( see def ault_atm_struct.m)

spectraviewer.m - is a GUI to locate and plot spectral files. The code was created using Matlab's GUIDE tool, so some of the code has been automatically gener ated.

1/0: spectraviewer; $\quad \%$ to start in current working directory spectraviewer(mydir); \% to start in mydir

wave.m - is a GUI calculator to convert between wavelength, wavenumber, and frequency. The code was created using Matlab's GUIDE tool, so some of the code has been automatically generated.

1/0: wave; 


\section{A.1.2 BSAS Calculation Functions}

bsas setup.m - defines directory locations for spectra and files used by BSAS.

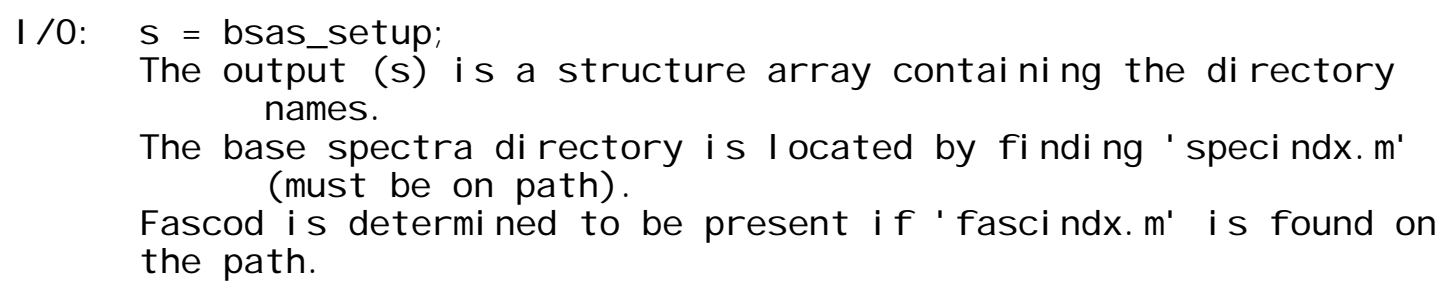

set_bsas_defaults.m- sets up the initial input data structure for the BSAS codè.

$$
\text { 1/0: } \begin{aligned}
& \text { in = set bsas defaults; } \\
& \text { in is the BSAS data structure }
\end{aligned}
$$

set bsas instrmodel defaults.m- sets up the input/output data structure used by dialsñr bsas calcic.m (for description of variables, see comments in di alsnr_bsàs_calc.m)

$$
\text { 1/0: model = set_bsas_instrmodel_defaults; }
$$

dialsnr_bsas_calc.m- calculates SNR and related parms for CW and pulsed lidar. Evalüates the instrumental noise for a CW or pulsed lidar system. The model includes the effects of shot noise, background noise, Johnson noise, detector noise, and speckle noise.

I/0: i n = dialsnr_bsas_calc(in)

\section{A.1.3 BSAS Fascode Functions}

fascod sas.m - is a function that sets up the input files to execute fascod $3 p$ directily from Matlab. It also reads the output files from fascod3p and returns the at mospheric spectra.

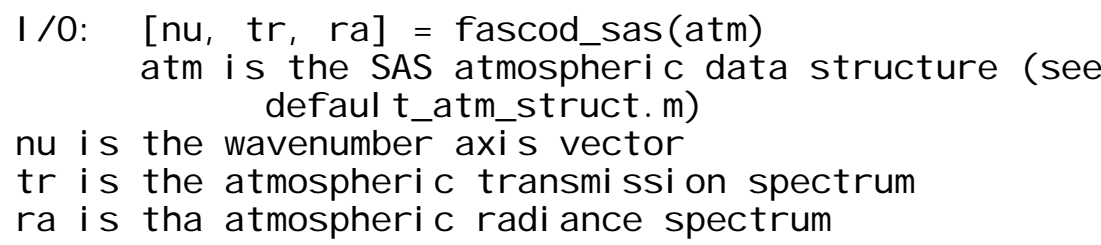

default_atm_struct.m - sets up default atm structure for fascod_sas.

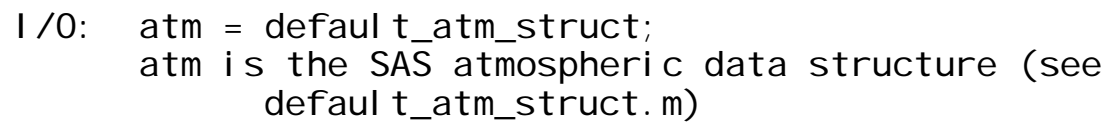

fascod sas defaults 0 . m - sets the default input card values for executing f ascod_sas from mat Tab. 
1/0: none - this code is evaluated directly inside fascod_sas using the Matlab'eval' function.

at mos_stats_sas.m - is a function to compute stats for at mospheric realiżations and make plots.

I/0: atmos_stats_sas(at mf i I ename, nat ms)

at mosfillename is the base filename for the atmos realizations

nat ms is the number of atmospheres (eg 100 is 0000 to 0099 )

h2ovaporpressure.m - returns vapor pressure of water at temperature temp (K). Vapor pressure of ice/water as function of temperature is taken from $p$. $6-10$ of the CRC handbook of chemistry and physics.

1/0: $\begin{aligned} & p=\text { h2ovaporpressure(temp) } \\ & \text { temp is the temperature in (K) } \\ & p \text { is the pressure in } P a\left(N / m^{\wedge} 2\right)\end{aligned}$

\section{A.1.4 BSAS Supporting Functions}

Bphoton.m - is the PIanck function for blackbody radiance spectral density in (photons/sec)/(cm^2)/sr/(cm^-1).

I/0: $\quad v=$ Bphoton( nu, t emp)

nu is the wavenumber in $\mathrm{cm}^{\wedge}$ - 1

temp is the temperature in $k$

cleanstr.m - removes underscore characters from a string - useful before passing to graph titles.

1/0: strout $=$ cleanstr(strin)

strin is the input string

strout is the output string

get_spectrum.m - reads in.tet. dat. xy or. spc spectral files.

1/0: [nu, v] = get spectrum(spectrumname)

spectrumname is the filename with.txt, dat, .xy, or. spc extension

. txt, dat, and. xy are all two col umn tab delimited asci i format . $S P C$ is a binary SPC file format (as used by GRAMS-32 software)

returns the wavenumber ( $x$-variable) as nu and the data as v

I LSconv.m - is an instrument I ine shape convolution code that performs a convolution of vkernel with vdata with vkernel normalized to have a sum of 1. 0 .

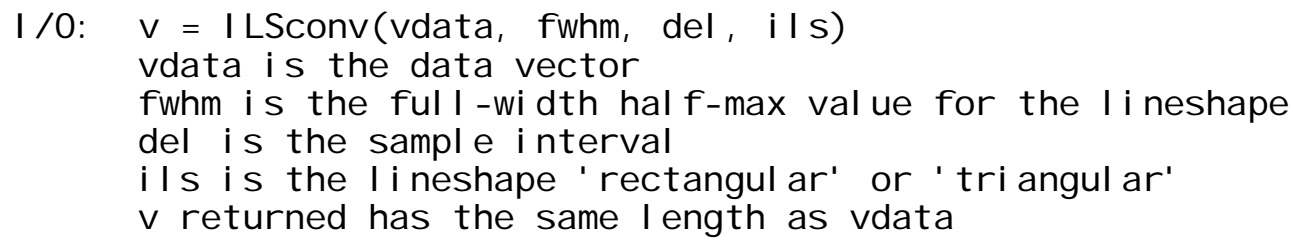

inputdlg alt.m - is modified version of internal function inputdlg.m in which the width of the modal pop-up GUl was reduced. 
I/0: see Matlab help for inputdlg.m

relpath.m - obtains relative path to subdir from maindir.

1/0: $\quad$ do = relpath(maindir, subdir)

maindir is the main directory

subdir is the sub-directory

do is the relative path between the main and sub directories

sinputdlg.m - gui to input several numeric variables contained in data

structure.

1/0: $\quad s=$ sinputdlg(s,sfields, slabels, titlestr)

$s=$ input/output data structure

sfields = list of field names to be input

slabels $=$ Iabels for each field i nput prompt

titlestr = title of dialog box

SpecReadSPC.m - reads in bi nary (GRAMS . spc) format files.

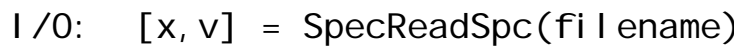

filename is the fil ename

returns wavenumber vector in $x$ and spectrum in $v$

SpecReadTxt.m - reads in two-column asci i format files files (spectra).

I/0: $[x, v]=\operatorname{SpecReadTxt}(f i \mid$ ename $)$

filename is the filename

returns wavenumber vector in $x$ and spectrum in $v$

SpecReadASP.m - reads, asp text format from I RASSOC/QASOFT database

I/0: $[x, v]=\operatorname{SpecReadASP}(f i \mid$ ename)

fil ename is the fil ename

returns wavenumber vector in $x$ and spectrum in $v$

SpecWritespc.m - writes a. spc (bi nary) format spectrum.

1/0: SpecWriteSpc(fil ename, $x, v)$

filename is the fil ename

$x$ is the wavenumber vector (or $x$ axis values)

$v$ is the spectral vectr (or y axis values

specWriteTxt.m - writes a two col umn (tab separated) format spectrum.

1/0: SpecWriteTxt ( f i I ename, x, v)

filename is the filename

$x$ is the wavenumber vector (or $x$ axis values)

$v$ is the spectral vectr (or y axis values

make_exp_form_str.m - converts v to a string using FORTRAN E style formatting

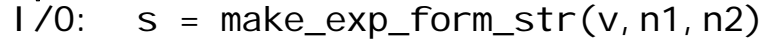

$v$ is the number to be formatted

nl is the total length of the formatted string

n2 is the number of digits to the left of the decimal pt 


\section{A.1.5 BSAS Aerosol Functions}

default _bsasaero_struct.m - default_bsasaero_struct - sets up default aerosol struct $\bar{f}$ or BSAS

1/0: aero = default_bsasaero_struct;

then run: aero = aerocs $($ aero);

aerocs.m - computes Mi e/ Tmatrix avg cross sections for a given particle size distribution. aerocs.m calls the functions ndistr3.m and Mie.m / tmatrix.m.

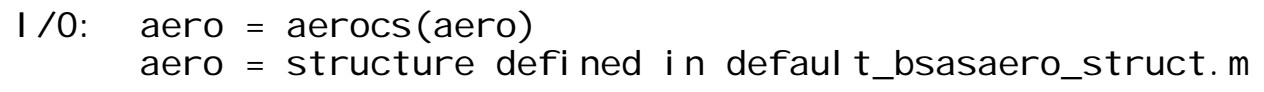

Mi e.m - Mie.m performs computation of Mie Efficiencies for given complex refractive-index ratio $m=m^{\prime}+i m^{\prime \prime}$ and size parameter $x=k 0 * a$, where $k 0=$ wave number i n ambient medium, a=sphere radius, using complex Mie coefficients an and bn for $n=1$ to nmax (Bohren and Huffman 1983) BEWI: TDD122, p. 103,119122,477. Result: $m^{\prime}, m^{\prime \prime}, x$, efficiencies for extinction (qext), scattering (qsca), absorption (qabs), backscattering ( $q b)$, asymmetry parameter ( as $y=<$ costet $a>$ ) and (qratio=qb/qsca). Uses the function "Mie abcd" for an and bn, for $n=1$ to nmax. This code was obtained from a report by C. Matzler $(2002)$.

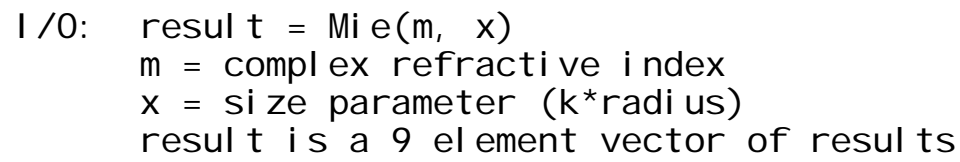

Mi e_abcd.m - computes a matrix of Mi e coefficients, $a_{-} n_{,} b_{-} n_{,} c_{-} n, d_{-} n$, of ordērs $n=1$ to nmax, complex refractive index $m=m^{\prime}+i m^{\prime \prime}$, and ${ }^{-}$si ze ${ }^{-}$parameter $x=k 0^{*} a$, where $k 0=$ wave number in the ambient medium, a =sphere radius; $p .100$, 477 i n Bohren and Huffman (1983). This code was obtained from a report by $C$. Matzler (2002).

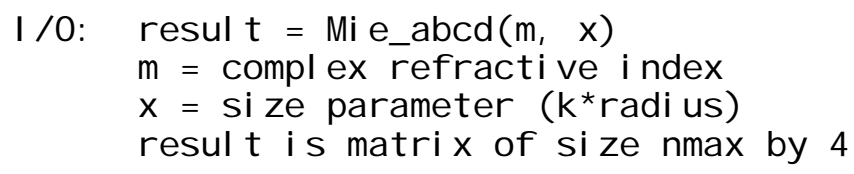

ndistr 3.m - computes the prob dens function for 6 different size distribution function definitions taken from Mi shchenko et al (2002). Distributions supported include:

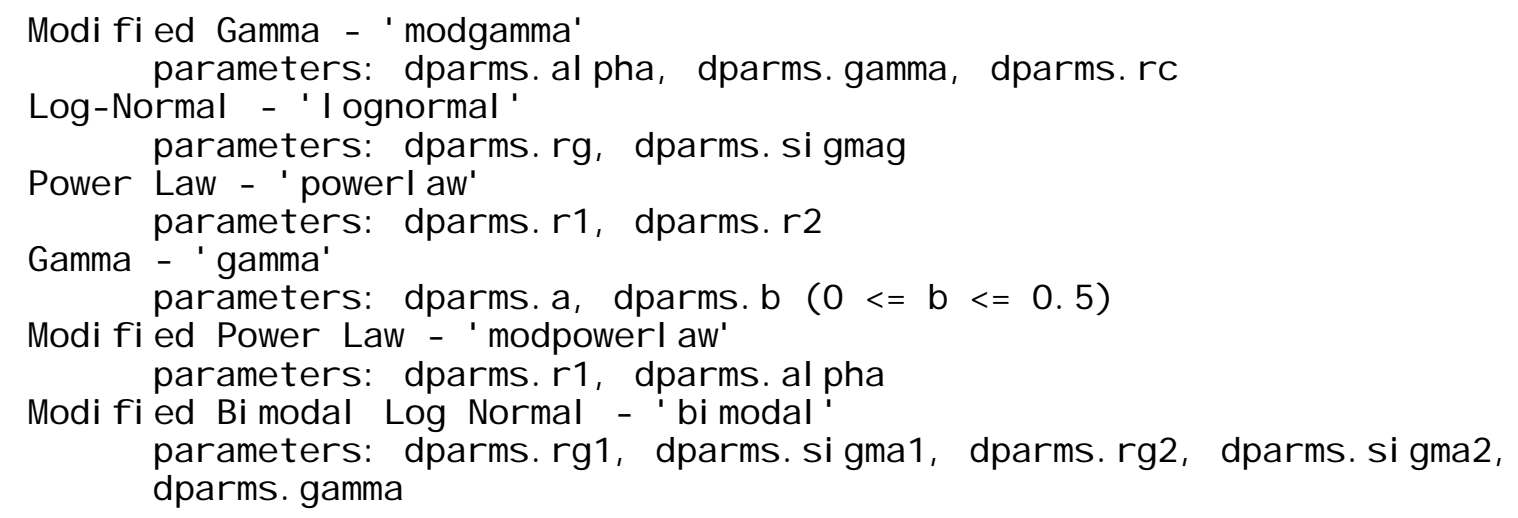


1/0: [n, reff, veff, Aeff] =ndistr3(r,w, dtype, dparms) where

$r$ is a vector of abscissas (x-axis of PDF)

w is a vector of weights for gauss quadrature (see gaussleg.m and ngauss (eg. m)

dtype is ' modgamma', 'I ognormal', 'gamma', 'powerlaw', ' modpowerlaw', or 'bi modal'

dparms is a struct with parms for each distr (def above)

$n$ is the PDF defined over the given $r^{\prime} s$

gaussleg.m - gaussleg.m computes the weights and abscissas for Gauss-Legendre quadrature integration. Adapted from "Numerical Recipes in $C$, 2nd edition $p$. 152.

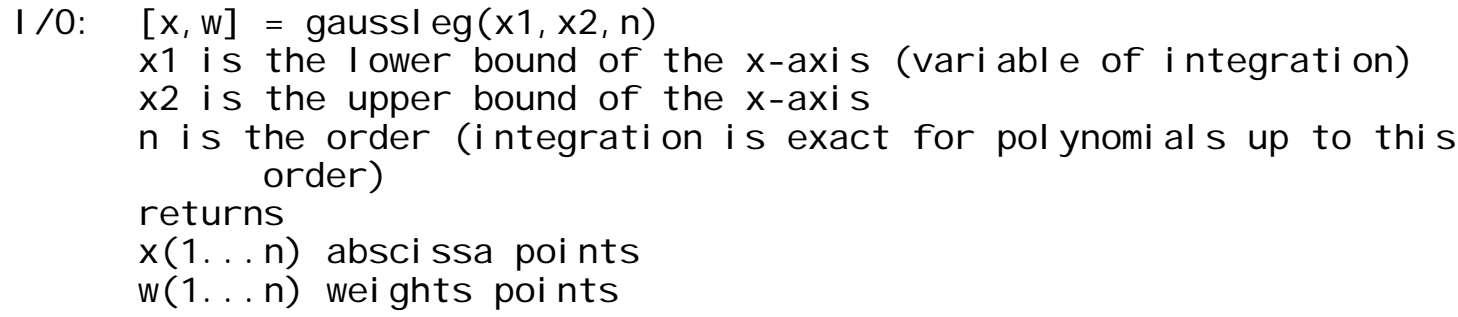

The integral of $f(x)$ from $x 1$ to 02 is then estimated by $\operatorname{sum}(f(x)$. * w ). Example:

$[x, w]=$ gaussleg $(0, p i, 4) ; \operatorname{sum}(\sin (x), * w)$

returns 2.000 (exact answer is 2)

ngaussleg.m - sets up points and weights for gauss-I egendre quadrature over a number of sub-intervals. Calls gaussleg.m to set up gauss quadrature points and weights for each subinterval ie. xv(1) to xv(2), xv(2) to xv(3) etc.

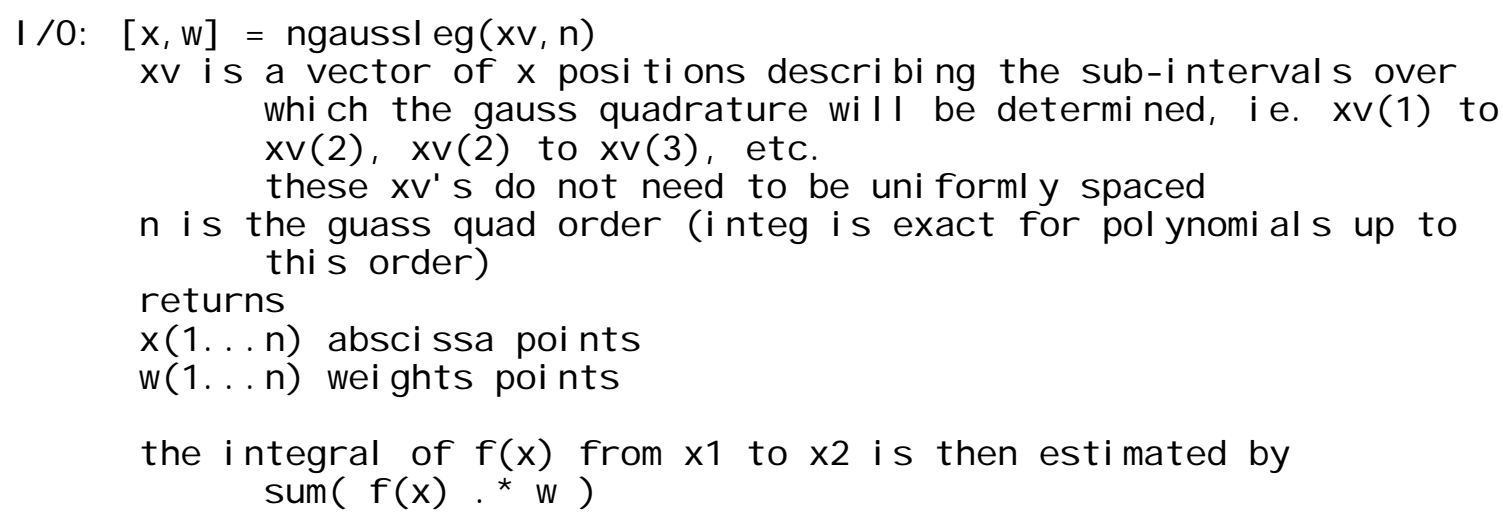


beta_rayl.m - Calculations of the Rayleigh scattering cross-section and

volume scattering coefficient - replicates work by Bucholtz (1995) derivation

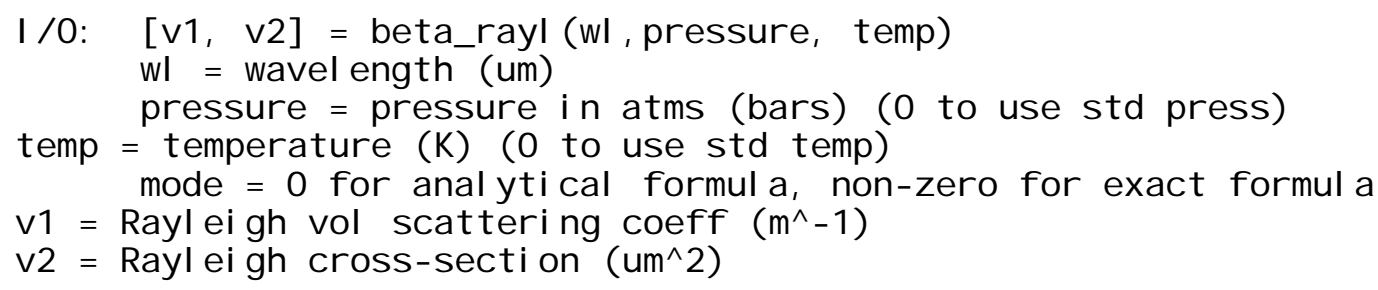

beta aero.m - Calculations of the natural aerosol volume scattering

coefficient - replicates work by Warren et al. (2004) this uses common

formula also described in Measures (1984, p. 143)

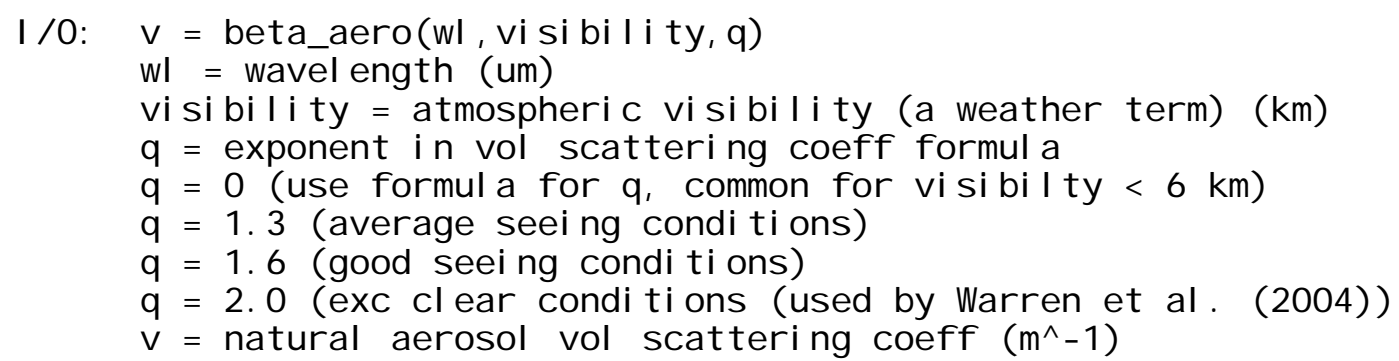

\section{A.2 References}

Bohren CF and DR Huffman. 1983. Absorption and Scattering of Light by Small Particles. John Wiley and Sons, New York.

Bucholtz A. 1995. "Rayleigh-scattering calculations for the terrestrial atmosphere." Appl Opt

34(15):2765-2773.

Matzler C. 2002. MATLAB Functions for Mie Scattering and Absorbance. Universitas Bernensis, Bern, Switzerland.

Measures RM. 1984. Laser Remote Sensing. John Wiley and Sons, New York.

Mishchenko MI, LD Travis and AA Lacis. 2002. Scattering, Absorption, and Emission of Light by Small Particles. Cambridge University Press, Cambridge (UK).

Warren JW, ME Thomas, EW Rogala, A Maret, CA Schumacher and A Diaz. 2004. "Systems Engineering Tradeoffs for a Bio-Aerosol Lidar Referee System.” Proceedings of SPIE 5416:202-215. 
A. 18 